TRANSACTIONS OF THE

AMERICAN MATHEMATICAL SOCIETY

Volume 353, Number 11, Pages 4497-4528

S 0002-9947(01)02719-2

Article electronically published on June 14, 2001

\title{
GROUND STATES AND SPECTRUM OF QUANTUM ELECTRODYNAMICS OF NONRELATIVISTIC PARTICLES
}

\author{
FUMIO HIROSHIMA
}

\begin{abstract}
A system consisting of finitely many nonrelativistic particles bound on an external potential and minimally coupled to a massless quantized radiation field without the dipole approximation is considered. An ultraviolet cut-off is imposed on the quantized radiation field. The Hamiltonian of the system is defined as a self-adjoint operator in a Hilbert space. The existence of the ground states of the Hamiltonian is established. It is shown that there exist asymptotic annihilation and creation operators. Hence the location of the absolutely continuous spectrum of the Hamiltonian is specified.
\end{abstract}

\section{INTRODUCTION}

In this paper we consider the Hamiltonian $H$ (the Pauli-Fierz Hamiltonian 12 , 43 ) describing a system of $N$-nonrelativistic particles (electrons) bound on an external potential and minimally coupled to a massless quantized radiation field (massless photon) without the dipole approximation. The radiation field is quantized in the Coulomb gauge, on which we impose an ultraviolet cut-off. In this paper we assume that (see Hypothesis 1, 2, 3 and 4 for details): (1) coupling constant $e$ is such that $0 \leq|e|<e_{0}$ with some $e_{0}>0$; (2) the ultraviolet cut-off satisfies mathematically convenient conditions; (3) the external potential is of the form: a quadratic potential plus a certain multiplication operator. Then we show the following facts: (i) the ground states of $H$ exist; (ii) asymptotic annihilation and creation operators exist; (iii) the absolutely continuous spectrum of $H$ is $[G, \infty$ ) with some $G$. A point we want to emphasize in this paper is to take no dipole approximation and to handle the massless photon.

The $N$-particles are governed by Hamiltonian $H_{\mathrm{el}}$ :

$$
H_{\mathrm{el}}:=-\frac{1}{2} \sum_{j=1}^{N} \Delta_{j}+V_{\mathrm{ex}}
$$

in $L^{2}\left(\mathbb{R}^{d N}\right)$, where $\Delta_{j}$ denotes the Laplacian in $L^{2}\left(\mathbb{R}^{d}\right)$, and $V_{\text {ex }}$ an external potential. Let $\mathcal{F}$ be a Boson Fock space. $H$ is a linear operator in $\mathcal{H}:=L^{2}\left(\mathbb{R}^{d N}\right) \otimes \mathcal{F}$, and is given by

$$
H:=\frac{1}{2} \sum_{j=1}^{N}\left(P^{j} \otimes I-e A\left(x^{j}\right)\right)^{2}+V_{\mathrm{ex}} \otimes I+I \otimes H_{\mathrm{f}} .
$$

Received by the editors August 14, 1998 and, in revised form, March 17, 2000.

1991 Mathematics Subject Classification. Primary 81Q10, 81U20, 47B15.

This work was supported in part by Japan Society for the Promotion of Science (JSPS). 
Here $P^{j}:=\left(p_{1}^{j}, \ldots, p_{d}^{j}\right)$ with $p_{\mu}^{j}:=-i \nabla_{x_{\mu}^{j}}, H_{\mathrm{f}}$ the free Hamiltonian in $\mathcal{F}$, and $A\left(x^{j}\right)$ the quantized radiation field.

The dipole approximation of $H$, say $H_{\text {dip }}$, is defined by $H$ with $A\left(x^{j}\right)$ replaced by $A(0)$. A. Arai has shown in a series of papers [5, 6, 7, , 8, 9] that $H_{\text {dip }}$ with a quadratic potential is exactly solvable, i.e., (1) there exists a unique ground state of $H_{\text {dip }}$; (2) the spectrum of $H_{\text {dip }}$ consists of point spectrum $\{E\}$ and purely absolutely continuous spectrum, $[E, \infty)$; (3) a wave operator exists and it is asymptotically complete. The analysis of $H_{\text {dip }}$ has been expanded to the case where $V_{\text {ex }}=$ a quadratic potential + some potentials, e.g. [42]. Spin Boson Hamiltonians regarded as simplified versions of $H_{\mathrm{dip}}$ [30] are studied in [4, 11, 29, 41]. In recent works [13, 14], without the dipole approximation, V. Bach, J. Fröhlich, and I. M. Sigal investigate a resonance (the instability of excited states) and the location of the purely absolutely continuous spectrum of $H$ by a renormalization group technique. In the case where each particle has spin, $H$-stability of the Pauli-Fierz Hamiltonian is investigated in [16, 17]. In [18, 19], the spectrum of $H\left(V_{\mathrm{ex}}=0\right)$ with a fixed total momentum (a polaron model) is studied. E. Lieb and M. Loss give an estimate of the bottom of the spectrum of $H$ in [37. In [28, the multiplicity of the ground state of $H$ is established by the Perron-Frobenius argument with the help of a functional integral of $e^{-t H}$ [24]. Moreover, it is proven in [27] that $H$ is essentially self-adjoint on some domain for arbitrary $e \in \mathbb{R}$ in the case $N=1$ in terms of the functional integral.

Throughout this paper we assume that

$$
V_{\mathrm{ex}}=V_{\mathrm{osc}}+V
$$

where $V$ is a multiplication operator, and $V_{\text {osc }}(x):=(1 / 2) \sum_{j=1}^{N}\left|x^{j}\right|^{2}$. By a momentum lattice approximation [11, 13, 19, 21, 38] we shall establish that there exists a ground state $\Omega_{\mathrm{g}}$ of $H$. Moreover, we shall show that if the ground state of $H_{\mathrm{el}}$ is unique, then $\Omega_{\mathrm{g}}$ strongly converges to a unique ground state of $H_{\mathrm{el}} \otimes I+I \otimes H_{\mathrm{f}}$ as $e \rightarrow 0$. Next we shall obtain that there exist strong limits of annihilation and creation operators, which we call asymptotic annihilation and creation operators, respectively [3. Note that in [1, 2, 31, 32, 33, 34, 35, 36, asymptotic annihilation and creation operators are studied for models with massive quantized fields. Algebraic relations between the asymptotic annihilation operators, the asymptotic creation operators, and $H$ are clarified. The asymptotic creation operators and $\Omega_{\mathrm{g}}$ provide for asymptotic incoming and outgoing Fock spaces. We write them as $\mathcal{F}_{-}$ and $\mathcal{F}_{+}$, respectively. We shall see that $\mathcal{F}_{ \pm}$are closed subspaces in $\mathcal{H}$, and that $H$ is reduced by $\mathcal{F}_{ \pm}$. We define an incoming wave operator and an outgoing one, $W_{-}: \mathcal{F} \rightarrow \mathcal{F}_{-}$and $W_{+}: \mathcal{F} \rightarrow \mathcal{F}_{+}$, respectively, which implement unitary equivalences $\mathcal{F}_{ \pm} \cong \mathcal{F}$ and $H\left\lceil\mathcal{F}_{ \pm} \cong H_{\mathrm{f}}-G\right.$. Hence we shall specify the location of the absolutely continuous spectrum of $H$. Taking no dipole approximation and handling massless photon are crucial for showing the existence of $\Omega_{\mathrm{g}}$, and for constructing the asymptotic annihilation and creation operators, respectively. The presence of $V_{\text {osc }}$ in $H$ enables us to avoid such troubles. Roughly speaking, $H_{\mathrm{el}} \otimes I+I \otimes H_{\mathrm{f}}$ plays a similar role as the number operator does in [21, 38].

An outline of this paper is as follows: in Section 2, we give the definition of $H$ and fundamental inequalities for later use. Furthermore we introduce Hypothesis 1, 2 and 3. In Section 3, we prove the existence of the ground states of $H$. In Section 4, introducing Hypothesis 4, we discuss the existence of the asymptotic annihilation and creation operators, and show the algebraic relations between them. 
Constructing $\mathcal{F}_{ \pm}$, we give the location of the absolutely continuous spectrum of $H$. Section 5 is devoted to investigating a Hamiltonian with spin, and Section 6 concluding remarks.

\section{Definition of a Hamiltonian}

2.1. Fundamental notation and definitions. Let us begin with definitions and expositions of notation used throughout this paper. For Hilbert space $\mathcal{K}$, the associated norm and scalar product are denoted by $\|f\|_{\mathcal{K}}$ and $(f, g)_{\mathcal{K}}, f, g \in \mathcal{K}$, respectively. Here $(f, g)_{\mathcal{K}}$ is linear in $g$ and antilinear in $f$. Unless confusion arises we omit $\mathcal{K}$ in $(\cdot, \cdot)_{\mathcal{K}}$ and $\|\cdot\|_{\mathcal{K}}$. The domain of operator $T$ is denoted by $D(T)$ and we set $C^{\infty}(T):=\bigcap_{n=1}^{\infty} D\left(T^{n}\right)$. We denote the spectrum, the absolutely continuous spectrum, the discrete spectrum and the point spectrum of $S$ by $\sigma(S), \sigma_{\mathrm{ac}}(S), \sigma_{\mathrm{d}}(S)$ and $\sigma_{\mathrm{p}}(S)$, respectively, and put $G(S):=\inf \sigma(S)$.

2.2. Perturbation of harmonic oscillators. A $d N$-dimensional harmonic oscillator is defined by

$$
H_{\mathrm{osc}}:=-\frac{1}{2} \sum_{j=1}^{N} \Delta_{j}+V_{\mathrm{osc}},
$$

which is essentially self-adjoint on $C_{0}^{\infty}\left(\mathbb{R}^{d N}\right)$, the set of infinite times differentiable functions with a compact support in $\mathbb{R}^{d N}$. The Hamiltonian of the particles is given by

$$
H_{\mathrm{el}}:=H_{\mathrm{osc}}+V
$$

Lemma 2.1. There exist constants $\alpha, \beta, \gamma, \delta>0$ so that, for $f \in D\left(H_{\mathrm{osc}}\right)$,

$$
\begin{aligned}
& \left\|-\frac{1}{2} \sum_{j=1}^{N} \Delta_{j} f\right\| \leq \alpha\left\|H_{\mathrm{osc}} f\right\|+\beta\|f\|, \\
& \left\|V_{\mathrm{osc}} f\right\| \leq \gamma\left\|H_{\mathrm{osc}} f\right\|+\delta\|f\| .
\end{aligned}
$$

Proof. Let $f \in C_{0}^{\infty}\left(\mathbb{R}^{d N}\right)$. Let $b_{\mu}^{j}:=\left(x_{\mu}^{j}+i p_{\mu}^{j}\right) / \sqrt{2}$ and $b_{\mu}^{\dagger j}:=\left(x_{\mu}^{j}-i p_{\mu}^{j}\right) / \sqrt{2}$, $\mu=1, \ldots, d, j=1, \ldots, N$. Then $H_{\mathrm{osc}}=\sum_{j=1}^{N} \sum_{\mu=1}^{d} b_{\mu}^{\dagger j} b_{\mu}^{j}+1 / 2,(-1 / 2) \sum_{j=1}^{N} \Delta_{j}=$ $(1 / 4) \sum_{j=1}^{N} \sum_{\mu=1}^{d}\left(b_{\mu}^{j}-b_{\mu}^{\dagger j}\right)^{2}$, and $V_{\text {osc }}=(1 / 4) \sum_{j=1}^{N} \sum_{\mu=1}^{d}\left(b_{\mu}^{j}+b_{\mu}^{\dagger j}\right)^{2}$. Using $\left[b_{\mu}^{i}, b_{\nu}^{\dagger j}\right]$ $=\delta_{\mu, \nu} \delta_{i, j},\left[b_{\mu}^{i}, b_{\nu}^{j}\right]=0,\left[b_{\mu}^{\dagger i}, b_{\nu}^{\dagger j}\right]=0$, on $C_{0}^{\infty}\left(\mathbb{R}^{d N}\right)$, one finds $\alpha, \beta, \gamma, \delta$ as in (2.1) and (2.2). Since $\sum_{j=1}^{N} \Delta_{j}$ and $V_{\text {osc }}$ are closed operators, (2.1) and (2.2) extend to $f \in D\left(\sum_{j=1}^{N} \Delta_{j}\right)$ and $f \in D\left(V_{\text {osc }}\right)$, respectively.

Suppose that $V$ is infinitesimally small with respect to $\sum_{j=1}^{N} \Delta_{j}$. Then (2.1) implies that $V$ is also infinitesimally small with respect to $H_{\text {osc }}$. Hence the KatoRellich theorem [39] Theorem X.12] yields that $H_{\mathrm{el}}$ is self-adjoint on $D\left(H_{\mathrm{osc}}\right)$, bounded below, and essentially self-adjoint on any core for $H_{\mathrm{osc}}$. We see that, for sufficiently small $\epsilon>0$, there exists $b(\epsilon)>0$ so that

$$
\left\|H_{\mathrm{osc}} f\right\| \leq\left(\left\|H_{\mathrm{el}} f\right\|+(b(\epsilon)+\epsilon \beta)\|f\|\right) /(1-\alpha \epsilon) \text { for } f \in D\left(H_{\mathrm{osc}}\right) .
$$


This means that, for $f \in D\left(H_{\mathrm{osc}}\right)$,

$$
\begin{gathered}
\left\|-\frac{1}{2} \sum_{j=1}^{N} \Delta_{j} f\right\| \leq \frac{\alpha}{1-\alpha \epsilon}\left\|H_{\mathrm{el}} f\right\|+\left(\frac{\alpha(b(\epsilon)+\epsilon \beta)}{1-\alpha \epsilon}+\beta\right)\|f\|, \\
\left\|V_{\mathrm{osc}} f\right\| \leq \frac{\gamma}{1-\alpha \epsilon}\left\|H_{\mathrm{el}} f\right\|+\left(\frac{\gamma(b(\epsilon)+\epsilon \beta)}{1-\alpha \epsilon}+\delta\right)\|f\| .
\end{gathered}
$$

Since $V$ is infinitesimally small with respect to $\sum_{j=1}^{N} \Delta_{j}$, it is also infinitesimally small in the sense of form. Then it follows that, for sufficiently small $0<\epsilon^{\prime}$, there exists $a\left(\epsilon^{\prime}\right)>0$ so that, for $f \in D\left(H_{\mathrm{osc}}\right)$,

$$
\left(f, H_{\mathrm{osc}} f\right)=\left(f, V_{\mathrm{osc}} f\right)+\left(f,-\frac{1}{2} \sum_{j=1}^{N} \Delta_{j} f\right) \leq \frac{1}{1-\epsilon^{\prime}}\left(f, H_{\mathrm{el}} f\right)+\frac{a\left(\epsilon^{\prime}\right)}{1-\epsilon^{\prime}}\|f\| .
$$

\subsection{Quantized radiation fields and Hamiltonians. Let}

$$
W:=\underbrace{L^{2}\left(\mathbb{R}^{d}\right) \oplus \ldots \oplus L^{2}\left(\mathbb{R}^{d}\right)}_{d-1} .
$$

Boson Fock space $\mathcal{F}$ is the Hilbert space completion of the infinite direct sum of the symmetric tensor algebra over $W$, i.e., $\mathcal{F}:=\bigoplus_{n=0}^{\infty} \mathcal{F}^{(n)}$, where $\mathcal{F}^{(0)}:=\mathbb{C}$, and $\mathcal{F}^{(n)}$ is defined by the $n$-fold symmetric tensor product of $W$ :

$$
\mathcal{F}^{(n)}:=\underbrace{W \otimes_{s} \cdots \otimes_{s} W}_{n}, \quad n \geq 1
$$

We write $\Phi \in \mathcal{F}$ as $\Phi=\left\{\Phi_{0}, \Phi_{1}, \Phi_{2}, \ldots\right\} \in \mathcal{F}^{(0)} \oplus \mathcal{F}^{(1)} \oplus \mathcal{F}^{(2)} \oplus \cdots$, and the bare vacuum, $\Omega$, of $\mathcal{F}$ is defined by $\Omega:=\{1,0,0, \ldots\}$. The annihilation operator, $a(F), F \in W$, and the creation operator, $a^{\dagger}(G), G \in W$, are defined in the usual way [3] and are linear in $F, G$, respectively. Put $a^{r}(f):=a\left(\oplus_{s=1}^{d-1} \delta_{r s} f\right)$ and $a^{\dagger r}(f):=$ $a^{\dagger}\left(\oplus_{s=1}^{d-1} \delta_{r s} f\right)$ for $f \in L^{2}\left(\mathbb{R}^{d}\right)$. $a^{\sharp r}$ stands for $a^{r}$ or $a^{\dagger r}$. We use the set $\mathcal{F}^{\infty}$ of vectors $\Psi=\left\{\Psi_{n}\right\}_{n=0}^{\infty}$ in $\mathcal{F}$ with $\Psi_{n}=0$ except for finitely many $n$ 's. Operators $a^{\sharp r}(f)$ leave $\mathcal{F}^{\infty}$ invariant and satisfy the canonical commutation relations on $\mathcal{F}^{\infty}$ :

$\left[a^{r}(f), a^{\dagger s}(g)\right]=\delta_{r s}(\bar{f}, g), \quad\left[a^{\sharp r}(f), a^{\sharp s}(g)\right]=0, \quad f, g \in L^{2}\left(\mathbb{R}^{d}\right), \quad r, s=1, \ldots, d-1$, where $\bar{f}$ denotes the complex conjugate of $f$. Moreover, for $\Psi_{1}, \Psi_{2} \in \mathcal{F}^{\infty}$,

$$
\left(\Psi_{1}, a^{r}(f) \Psi_{2}\right)=\left(a^{\dagger r}(\bar{f}) \Psi_{1}, \Psi_{2}\right), \quad f \in L^{2}\left(\mathbb{R}^{d}\right), \quad r=1, \ldots, d-1 .
$$

By this we see that $a^{\sharp r}(f)$ are closable operators. We write their closures as the same symbols. $a^{\sharp r}(k)$ denotes a "formal" operator-valued distribution associated with $a^{\sharp r}(f): a^{\sharp r}(f)=\int a^{\sharp r}(k) f(k) d k$. The free Hamiltonian of $\mathcal{F}$ reads

$$
H_{\mathrm{f}}:=\sum_{r=1}^{d-1} \int \omega(k) a^{\dagger r}(k) a^{r}(k) d k,
$$

where $\omega(k):=|k|$ describes the kinetic energy of the photon of momentum $k \in \mathbb{R}^{d}$. $H_{\mathrm{f}}$ is a positive self-adjoint operator with

$$
\sigma\left(H_{\mathrm{f}}\right)=[0, \infty), \quad \sigma_{\mathrm{p}}\left(H_{\mathrm{f}}\right)=\{0\}, \quad \sigma_{\mathrm{ac}}\left(H_{\mathrm{f}}\right)=[0, \infty),
$$

where $\{0\}$ is non-degenerate and its associated unique eigenvector is $\Omega$ :

$$
H_{\mathrm{f}} \Omega=0 .
$$


The number operator, $N$, is defined by

$$
\begin{gathered}
N:=\sum_{r=1}^{d-1} \int a^{\dagger r}(k) a^{r}(k) d k \\
D(N):=\left\{\left\{\Psi_{n}\right\}_{n=0}^{\infty} \in \mathcal{F} \mid \sum_{n=0}^{\infty} n^{2}\left\|\Psi_{n}\right\|_{\mathcal{F}(n)}^{2}<\infty\right\} .
\end{gathered}
$$

Let $\mathcal{M}_{n}:=\left\{f \mid\|f\|_{n}:=\left\|(\sqrt{\omega})^{n} f\right\|_{L^{2}\left(\mathbb{R}^{d}\right)}<\infty\right\}$. In particular, we set

$$
\mathbf{M}_{4}:=\mathcal{M}_{-1} \cap \mathcal{M}_{0} \cap \mathcal{M}_{1} \cap \mathcal{M}_{2}, \quad \mathbf{M}_{2}:=\mathcal{M}_{-1} \cap \mathcal{M}_{0} .
$$

It is known that, if $f \in \bigcap_{k=-1}^{2 n} \mathcal{M}_{k}$, then $a^{\sharp r}(f)$ maps $D\left(H_{\mathrm{f}}^{n+1 / 2}\right)$ into $D\left(H_{\mathrm{f}}^{n}\right)$, and that

$$
\begin{gathered}
\left\|a^{\dagger r}(f) \Psi\right\| \leq\|f\|_{-1}\left\|H_{\mathrm{f}}^{1 / 2} \Psi\right\|+\|f\|_{0}\|\Psi\|, \\
\left\|a^{r}(f) \Psi\right\| \leq\|f\|_{-1}\left\|H_{\mathrm{f}}^{1 / 2} \Psi\right\|, \\
{\left[H_{\mathrm{f}}, a^{\dagger r}(g)\right] \Psi^{\prime}=a^{\dagger r}(\omega g) \Psi^{\prime},} \\
{\left[H_{\mathrm{f}}, a^{r}(g)\right] \Psi^{\prime}=-a^{r}(\omega g) \Psi^{\prime},} \\
\left\|\left[\left(H_{\mathrm{f}}+I\right)^{1 / 2}, a^{\sharp r}(g)\right] \Psi^{\prime}\right\| \leq \mathbf{k}\left(\|g\|_{1}+\|g\|_{2}\right)\left\|\left(H_{\mathrm{f}}+I\right)^{1 / 2} \Psi^{\prime}\right\|, \\
\left\|a^{\dagger r}(h) \Phi\right\| \leq\|h\|_{0}\left\|(N+I)^{1 / 2} \Phi\right\|, \quad\left\|a^{r}(h) \Phi\right\| \leq\|h\|_{0}\left\|N^{1 / 2} \Phi\right\|,
\end{gathered}
$$

where $\Psi \in D\left(H_{\mathrm{f}}^{1 / 2}\right), \Psi^{\prime} \in D\left(H_{\mathrm{f}}^{3 / 2}\right), \Phi \in D\left(N^{1 / 2}\right), f \in \mathbf{M}_{2}, g \in \mathbf{M}_{4}, h \in \mathcal{M}_{0}$, and $\mathbf{k}:=\int_{0}^{\infty} \sqrt{\lambda} /(\lambda+1)^{2} d \lambda /\left(2 \pi \sqrt{(2 \pi)^{d}}\right)$ (see [10]).

Lemma 2.2. Let $\Psi \in D\left(H_{\mathrm{f}}\right), g \in \mathbf{M}_{4}$ and $f \in \mathbf{M}_{2}$. Then

$$
\begin{aligned}
\left\|a^{\sharp r}(f) a^{\sharp s}(g) \Psi\right\| \leq & \mathbf{k}\left(\|f\|_{-1}+\|f\|_{0}\right)\left(\|g\|_{1}+\|g\|_{2}\right)\left\|\left(H_{\mathrm{f}}+I\right)^{1 / 2} \Psi\right\| \\
& +\left(\|f\|_{-1}+\|f\|_{0}\right)\left(\|g\|_{-1}+\|g\|_{0}\right)\left\|\left(H_{\mathrm{f}}+I\right) \Psi\right\| .
\end{aligned}
$$

Proof. From (2.6) and (2.7), for $\Psi \in D\left(H_{\mathrm{f}}^{3 / 2}\right)$, (2.9) follows. For any $\Psi \in D\left(H_{\mathrm{f}}\right)$, one can find $\Psi_{n} \in D\left(H^{3 / 2}\right)$ so that $\Psi_{n} \rightarrow \Psi$ and $H_{\mathrm{f}} \Psi_{n} \rightarrow H_{\mathrm{f}} \Psi$ strongly as $n \rightarrow \infty$. Since $a^{\sharp s}(g)$ is closed, (2.6) employs that $\Psi \in D\left(a^{\sharp s}(g)\right)$, and that $a^{\sharp s}(g) \Psi_{n}$ converges to $a^{\sharp s}(g) \Psi$. From the closedness of $a^{\sharp r}(f), a^{\sharp s}(g) \Psi \in D\left(a^{\sharp r}(f)\right)$ and (2.9) follows.

By Lemma 2.2, the canonical commutation relations of $a^{\sharp r}(f), f \in \mathbf{M}_{4}$, extend to those on $D\left(H_{\mathrm{f}}\right)$. The Hilbert space considered in this paper is

$$
\mathcal{H}:=L^{2}\left(\mathbb{R}^{d N}\right) \otimes \mathcal{F} \cong L^{2}\left(\mathbb{R}^{d N} ; \mathcal{F}\right) \equiv \int_{\mathbb{R}^{d N}}^{\oplus} \mathcal{F} d x .
$$

Here $L^{2}\left(\mathbb{R}^{d N} ; \mathcal{F}\right)$ denotes the set of $\mathcal{F}$-valued $L^{2}$-functions on $\mathbb{R}^{d N}$. The Hamiltonian reads

$$
H:=H(\hat{\rho}):=\frac{1}{2} \sum_{j=1}^{N}\left(P^{j} \otimes I-e A\left(x^{j}\right)\right)^{2}+\left(V+V_{\text {osc }}\right) \otimes I+I \otimes H_{\mathrm{f}},
$$

where $A\left(x^{j}\right):=A\left(\hat{\rho}, x^{j}\right):=\left(A_{1}\left(\hat{\rho}, x^{j}\right), \ldots, A_{d}\left(\hat{\rho}, x^{j}\right)\right)$ is given by

$$
A_{\mu}(\hat{\rho}, x):=\sum_{r=1}^{d-1} \int d k\left\{\frac{\hat{\rho}(k) e_{\mu}^{r}(k) e^{-i k x}}{\sqrt{2 \omega(k)}} a^{\dagger r}(k)+\frac{\hat{\rho}(-k) e_{\mu}^{r}(k) e^{i k x}}{\sqrt{2 \omega(k)}} a^{r}(k)\right\},
$$


with $e^{r}:=\left(e_{1}^{r}, \ldots, e_{d}^{r}\right)$ such that $e^{r}(k) \cdot e^{s}(k)=\delta_{r, s}$ and $k \cdot e^{r}(k)=0$, a.e. $k \in \mathbb{R}^{d}$. Moreover, $\hat{\rho}(k):=(2 \pi)^{-d / 2} \int_{\mathbb{R}^{d}} \rho(s) e^{-i k s} d s$ is an ultraviolet cut-off. In what follows, for simplicity, we assume that $e>0$. We introduce the following hypotheses:

Hypothesis 1: We assume that $0 \leq e<e_{0}$, where $e_{0}$ is given in Subsection 2.4;

Hypothesis 2: Ultraviolet cut-off $\hat{\rho}$ is continuous with $\hat{\rho}(-k)=\overline{\hat{\rho}}(k)$ and satisfies that $\|\hat{\rho}\|_{n}<\infty, n=-3,-2,-1,0,1$. Moreover, $e_{\mu}^{r}$ satisfies that $e_{\mu}^{r} \in C^{\infty}\left(\mathbb{R}^{d} \backslash \mathcal{P}\right)$ for $\mu=1, \ldots, d, r=1, \ldots, d-1$, where $\mathcal{P} \subset \mathbb{R}^{d}$ is a set with Lebesgue measure zero;

Hypothesis 3: $V$ is infinitesimally small with respect to $\sum_{j=1}^{N} \Delta_{j}$.

For example, in the case where $d=3$, we can take $\mathcal{P}=\left\{k=\left(0,0, k_{3}\right) \in \mathbb{R}^{3} \mid k_{3} \in \mathbb{R}\right\}$. Under Hypothesis 3, $\sigma\left(H_{\mathrm{el}}\right)=\sigma_{\mathrm{d}}\left(H_{\mathrm{el}}\right)$. Assume Hypothesis 3. Then

$$
H_{0}:=H_{\mathrm{el}} \otimes I+I \otimes H_{\mathrm{f}}
$$

is self-adjoint on $\mathcal{D}_{0}:=D\left(H_{\mathrm{osc}} \otimes I\right) \cap D\left(I \otimes H_{\mathrm{f}}\right)$ and bounded below. We note that, on $\mathcal{D}_{0}$ (the Coulomb gauge condition),

$$
\sum_{\mu=1}^{d}\left(p_{\mu}^{j} \otimes I\right) A_{\mu}\left(x^{j}\right)=\sum_{\mu=1}^{d} A_{\mu}\left(x^{j}\right)\left(p_{\mu}^{j} \otimes I\right), \quad j=1, \ldots, N .
$$

Then we write $H$ as a perturbation of $H_{0}$, i.e., $H=H_{0}+e H_{\mathrm{I}}+e^{2} H_{\mathrm{II}}$, where

$$
\begin{gathered}
H_{\mathrm{I}}:=H_{\mathrm{I}}(\hat{\rho}):=-\sum_{j=1}^{N} \sum_{\mu=1}^{d}\left(p_{\mu}^{j} \otimes I\right) A_{\mu}\left(x_{j}\right), \\
H_{\mathrm{II}}:=H_{\mathrm{II}}(\hat{\rho}):=\frac{1}{2} \sum_{j=1}^{N} \sum_{\mu=1}^{d} A_{\mu}\left(x_{j}\right)^{2} .
\end{gathered}
$$

2.4. Self-adjointness and inequalities. It is convenient to give some estimates on $H_{0}, H_{\mathrm{I}}$ and $H_{\mathrm{II}}$. Generally, for self-adjoint operators $S$ and $T$ in $L^{2}\left(\mathbb{R}^{d N}\right)$ and $\mathcal{F}$, respectively, and for $\Psi \in D\left(S^{2} \otimes I\right) \cap D\left(I \otimes T^{2}\right)$, one sees that $\|S \otimes T \Psi\| \leq$ $\left(\left\|S^{2} \otimes I \Psi\right\|+\left\|I \otimes T^{2} \Psi\right\|\right) / \sqrt{2}$. Then using (2.3), (2.6), and (2.9), we see that, under Hypothesis 2 , for $\Psi \in \mathcal{D}_{0}$,

$$
\begin{gathered}
\left\|H_{\mathrm{I}} \Psi\right\| \leq A\left\|I \otimes H_{\mathrm{f}} \Psi\right\|+\tilde{B}\left\|-\frac{1}{2} \sum_{j=1}^{N} \Delta_{j} \otimes I \Psi\right\|+\tilde{C}\|\Psi\| \\
\leq A\left\|I \otimes H_{\mathrm{f}} \Psi\right\|+B\left\|H_{\mathrm{el}} \otimes I \Psi\right\|+C\|\Psi\|, \\
\left\|H_{\mathrm{II}} \Psi\right\| \leq \tilde{D}\left\|\left(I+I \otimes H_{\mathrm{f}}\right) \Psi\right\|+\tilde{E}\left\|\left(I+I \otimes H_{\mathrm{f}}\right)^{1 / 2} \Psi\right\| \leq D\left\|I \otimes H_{\mathrm{f}} \Psi\right\|+E\|\Psi\| .
\end{gathered}
$$

Here, with $\alpha, \beta, \epsilon, b(\epsilon)$ in (2.3),

$$
\begin{gathered}
A:=d(d-1) N\|\hat{\rho}\|_{-2}, \quad \tilde{B}:=d(d-1) N\left(2\|\hat{\rho}\|_{-2}+\|\hat{\rho}\|_{-1}\right), \quad \tilde{C}:=d(d-1) N\|\hat{\rho}\|_{-1} / 2, \\
B:=\frac{\alpha \tilde{B}}{1-\alpha \epsilon}, \quad C:=\tilde{B}\left(\frac{\alpha(b(\epsilon)+\epsilon \beta)}{1-\alpha \epsilon}+\beta\right)+\tilde{C}, \\
\tilde{D}:=d(d-1) N\left(\|\hat{\rho}\|_{-2}+\|\hat{\rho}\|_{-1}\right)^{2}, \quad \tilde{E}:=d(d-1) N \mathbf{k}\left(\|\hat{\rho}\|_{-2}+\|\hat{\rho}\|_{-1}\right)\left(\|\hat{\rho}\|_{0}+\|\hat{\rho}\|_{1}\right), \\
D:=E:=\tilde{D}+\tilde{E} / 2 .
\end{gathered}
$$


Let $\mathbf{g}=\inf \sigma\left(H_{\mathrm{el}}\right)$. We set $M_{\mathrm{I}}:=\max \{A+B,|\mathbf{g}| A+2|\mathbf{g}| B+C\}$ and $M_{\mathrm{II}}:=|\mathbf{g}| D+E$. Note that

$$
\begin{aligned}
& \left\|I \otimes H_{\mathrm{f}} \Psi\right\| \leq\left\|H_{0} \Psi\right\|+|\mathbf{g}|\|\Psi\|, \\
& \left\|H_{\mathrm{el}} \otimes I \Psi\right\| \leq\left\|H_{0} \Psi\right\|+2|\mathbf{g}|\|\Psi\| .
\end{aligned}
$$

Then for $\Psi \in \mathcal{D}_{0}$,

$$
\left\|H_{\mathrm{I}} \Psi\right\| \leq M_{\mathrm{I}}\left(\left\|H_{0} \Psi\right\|+\|\Psi\|\right), \quad\left\|H_{\mathrm{II}} \Psi\right\| \leq M_{\mathrm{II}}\left(\left\|H_{0} \Psi\right\|+\|\Psi\|\right) .
$$

Similarly, using (2.5) and (2.6), one can deduce inequalities of form versions; for $\Psi \in \mathcal{D}_{0}$,

$$
\begin{gathered}
\left|\left(\Psi, H_{\mathrm{I}} \Psi\right)\right| \leq M_{\mathrm{I}}^{\prime}\left(\Psi, H_{0} \Psi\right)+\alpha_{\mathrm{I}}\|\Psi\|^{2}, \\
\left|\left(\Psi, H_{\mathrm{II}} \Psi\right)\right| \leq M_{\mathrm{II}}^{\prime}\left(\Psi, H_{0} \Psi\right)+\alpha_{\mathrm{II}}\|\Psi\|^{2},
\end{gathered}
$$

where, with $\epsilon^{\prime}, a\left(\epsilon^{\prime}\right)$ in (2.5),

$$
\begin{gathered}
M_{\mathrm{I}}^{\prime}:=\sqrt{2} A\left(\frac{1}{1-\epsilon^{\prime}}+\frac{1}{2}\right), \quad \alpha_{\mathrm{I}}:=\sqrt{2} A\left(\frac{a\left(\epsilon^{\prime}\right)}{1-\epsilon^{\prime}}-\frac{\mathbf{g}}{2}\right), \\
M_{\mathrm{II}}^{\prime}:=\widetilde{B}\|\hat{\rho}\|_{-2} / 2, \quad \alpha_{\mathrm{II}}:=\widetilde{B}\left(\|\hat{\rho}\|_{-1}-2 \mathbf{g}\|\hat{\rho}\|_{-2}\right) / 4 .
\end{gathered}
$$

Thus we define $1 e_{0}$ announced in Hypothesis 1 by

$$
e_{0}:=\min \left\{\theta_{1}, \theta_{2} \mid \theta_{1} M_{\mathrm{I}}+\theta_{1}^{2} M_{\mathrm{II}}=1 / 2, \delta\left(\theta_{2}\right)=1, \theta_{1}>0, \theta_{2}>0\right\} .
$$

Here function $\delta(\cdot)$ is defined in (3.22), which is a monotone increasing function with $\delta(0)=0$. If $0 \leq e<e_{0}$, then $e M=e M_{\mathrm{I}}+e^{2} M_{\mathrm{II}}<1$. We set $M^{\prime}:=M_{\mathrm{I}}^{\prime}+e M_{\mathrm{II}}^{\prime}$. Thus in view of (2.13) and (2.14), for $\Psi \in \mathcal{D}_{0}$, it follows that

$$
\begin{aligned}
\left\|H_{0} \Psi\right\| & \leq(\|H \Psi\|+\|\Psi\|) /(1-e M) \\
\left\|H_{\mathrm{I}} \Psi\right\| & \leq 2 M_{\mathrm{I}}(\|H \Psi\|+\|\Psi\|) /(1-e M), \\
\left\|H_{\mathrm{II}} \Psi\right\| & \leq 2 M_{\mathrm{II}}(\|H \Psi\|+\|\Psi\|) /(1-e M), \\
\|H \Psi\| & \leq(e M+1)\left\|H_{0} \Psi\right\|+M\|\Psi\|, \\
(\Psi, H \Psi) & \leq\left(e M^{\prime}+1\right)\left(\Psi, H_{0} \Psi\right)+\left(e \alpha_{\mathrm{I}}+e^{2} \alpha_{\mathrm{II}}\right)\|\Psi\|^{2}, \\
\left(1-e M^{\prime}\right)\left(\Psi, H_{0} \Psi\right) & \leq(\Psi, H \Psi)+\left(e \alpha_{\mathrm{I}}+e^{2} \alpha_{\mathrm{II}}\right)\|\Psi\|^{2} .
\end{aligned}
$$

We have the following proposition.

Proposition 2.3. Assume that Hypothesis 1, 2 and 3 hold. Then $H$ is self-adjoint on $\mathcal{D}_{0}$ and bounded below. Moreover, it is essentially self-adjoint on any core for $H_{0}$.

Proof. Since $H_{0}$ is self-adjoint on $\mathcal{D}_{0}$ and $H_{\mathrm{I}}, H_{\mathrm{II}}$ are symmetric, from (2.13) the Kato-Rellich theorem yields the desired results.

Remark 2.4. The essential self-adjointness of $H$ for arbitrary $e \in \mathbb{R}$ is established in [27.

Remark 2.5. (1) For the proof of the self-adjointness of $H$ in Proposition 2.3, we do not need to assume that $\hat{\rho}$ and $e_{\mu}^{r}$ are continuous, and that $\|\hat{\rho}\|_{-3}<\infty$.

\footnotetext{
${ }^{1}$ Condition $\theta_{1} M_{\mathrm{I}}+\theta_{1}^{2} M_{\mathrm{II}}<1 / 2$ comes from a sufficient condition for $H$ to be self-adjoint and ensuring (3.11), and condition $\delta\left(\theta_{2}\right)<1$ from a proof of an overlap (Lemma 3.11].
} 
(2) Generally, for semibounded self-adjoint operators $K$ and $L$ in Hilbert space $\mathcal{L}$ so that $(\Psi, L \Psi)_{\mathcal{L}} \leq(\Psi, K \Psi)_{\mathcal{L}}, \Psi \in D(K) \subset D(L)$, we see that $(\Psi,|L| \Psi)_{\mathcal{L}} \leq$ $(\Psi,|K| \Psi)_{\mathcal{L}}+2|G(L)|\|\Psi\|_{\mathcal{L}}^{2}$. Then from (2.19) and (2.20) it follows that $D\left(\left|H_{0}\right|^{1 / 2}\right)=D\left(|H|^{1 / 2}\right)$.

Proposition 2.6. Assume that Hypothesis 1, 2 and 3 hold, and $\Psi \in D\left(\left|H_{0}\right|^{1 / 2}\right)$ and $\Phi \in D\left(H_{0}\right)$. Then there exist positive constants $\alpha_{k}, k=1, \ldots, 8$, so that

$$
\begin{aligned}
\left\|p_{\mu}^{j} \otimes I \Psi\right\| \leq & \alpha_{1}\left(\left\|\left|H_{0}\right|^{1 / 2} \Psi\right\|+\|\Psi\|\right), \\
\left\|\left|x^{j}\right| \otimes I \Psi\right\| \leq & \alpha_{2}\left(\left\|\left|H_{0}\right|^{1 / 2} \Psi\right\|+\|\Psi\|\right), \\
\left\|I \otimes H_{\mathrm{f}}^{1 / 2} \Psi\right\| \leq & \alpha_{3}\left(\left\|\left|H_{0}\right|^{1 / 2} \Psi\right\|+\|\Psi\|\right), \\
\left\|\left(p_{\mu}^{j}\right)^{2} \otimes I \Phi\right\| \leq & \alpha_{4}\left(\left\|H_{0} \Phi\right\|+\|\Phi\|\right), \\
\left\|\left(x_{\mu}^{j}\right)^{2} \otimes I \Phi\right\| \leq & \alpha_{5}\left(\left\|H_{0} \Phi\right\|+\|\Phi\|\right), \\
\left\|\left(x_{\mu}^{j} p_{\mu}^{j}\right) \otimes I \Phi\right\| \leq & \alpha_{6}\left(\left\|H_{0} \Phi\right\|+\|\Phi\|\right), \\
\left\|p_{\mu}^{j} \otimes H_{\mathrm{f}}^{1 / 2} \Phi\right\| \leq & \alpha_{7}\left(\left\|H_{0} \Phi\right\|+\|\Phi\|\right), \\
\left\|x^{j} \mid \otimes H_{\mathrm{f}}^{1 / 2} \Phi\right\| \leq & \alpha_{8}\left(\left\|H_{0} \Phi\right\|+\|\Phi\|\right), \\
& j=1, \ldots, N, \mu=1, \ldots, d .
\end{aligned}
$$

Proof. First we assume that $\Psi \in C_{0}^{\infty}\left(\mathbb{R}^{d N}\right) \widehat{\otimes} D\left(H_{\mathrm{f}}\right)$, where $\widehat{\otimes}$ means algebraic tensor product. Since $\left\|p_{\mu}^{j} \otimes I \Psi\right\|^{2}=\left(\Psi,\left(p_{\mu}^{j}\right)^{2} \otimes I \Psi\right)$, (2.21) follows from (2.5). Similarly (2.22) follows. (2.23) is trivial. (2.24) and (2.25) follow from (2.3) and (2.4). Since

$$
\begin{aligned}
\left\|\left(x_{\mu}^{j} p_{\mu}^{j}\right) \otimes I \Psi\right\|^{2} & =2 i\left(p_{\mu}^{j} \otimes I \Psi, x_{\mu}^{j} \otimes I \Psi\right)+\left(\left(p_{\mu}^{j}\right)^{2} \otimes I \Psi,\left(x_{\mu}^{j}\right)^{2} \otimes I \Psi\right) \\
& \leq 2\left\|p_{\mu}^{j} \otimes I \Psi\right\|\left\|x_{\mu}^{j} \otimes I \Psi\right\|+\left\|\left(p_{\mu}^{j}\right)^{2} \otimes I \Psi\right\|\left\|\left(x_{\mu}^{j}\right)^{2} \otimes I \Psi\right\|,
\end{aligned}
$$

(2.26) follows from (2.21) $,(2.22),(2.24)$ and (2.25). We see that $\left\|p_{\mu}^{j} \otimes H_{\mathrm{f}}^{1 / 2} \Psi\right\|^{2} \leq$ $\left\|\left(p_{\mu}^{j}\right)^{2} \otimes I \Psi\right\|\left\|I \otimes H_{\mathrm{f}} \Psi\right\|$. Thus (2.27) follows from (2.24). (2.28) is similar to (2.27). Because of the fact that $C_{0}^{\infty}\left(\mathbb{R}^{d N}\right) \widehat{\otimes} D\left(H_{\mathrm{f}}\right)$ is a core for $H_{0}$, (2.21) $-(2.28)$ extend to those on $D\left(H_{0}\right)$. In addition, since $D\left(H_{0}\right)$ is a form core for $\left|H_{0}\right|^{1 / 2},(2.21)-(2.23)$ extend to those on $D\left(\left|H_{0}\right|^{1 / 2}\right)$.

Proposition 2.7. Assume that Hypothesis 1, 2 and 3 hold. Then we have

$$
\mathbf{g} \leq G(H) \leq\left(1+e M^{\prime}\right) \mathbf{g}+e \alpha_{\mathrm{I}}+e^{2} \alpha_{\mathrm{II}} .
$$

Proof. Note that $G\left(H_{0}\right)=\mathrm{g}$. The right-hand side of (2.29) follows from (2.19). The left-hand side is due to the diamagnetic inequality $\sqrt{2}$ for the pair $\left(H_{0}, H\right)$. See [23], 24].

Thus from Proposition 2.7] it follows that $\lim _{e \rightarrow 0} G(H)=\mathbf{g}$.

\section{Existence of GRound states}

3.1. Overview. In this section, we establish the existence of the ground states of $H$. We apply the momentum lattice approximation used in [11, 13, 21, 38. Throughout section 3 we assume that Hypothesis 1, 2 and 3 hold. We first quickly see an overview of this section. We define $H^{m}:=H^{m}(\hat{\rho}), m>0$, by $H$ with $H_{\mathrm{f}}$ replaced by $\sum_{r=1}^{d-1} \int(\omega(k)+m) a^{\dagger r}(k) a^{r}(k) d k$. Let $\chi_{L}$ be the characteristic

\footnotetext{
${ }^{2}$ Unless using diamagnetic inequalities, we have from (2.20) $\left(1-e M^{\prime}\right) \mathbf{g} \leq G(H)+e \alpha_{\mathrm{I}}+e^{2} \alpha_{\mathrm{II}}$.
} 
function of $\left\{k \in \mathbb{R}^{d}|| k \mid<L\right\}$ and $\hat{\rho}_{L}:=\chi_{L} \hat{\rho}$. We can construct closed subspaces $\mathcal{F}_{a} \subset \mathcal{F}$ and self-adjoint operators $H_{a, L}^{m}$ with index $a>0$ so that $H_{a, L}^{m}$ uniformly converges to $H_{L}^{m}:=H^{m}\left(\hat{\rho}_{L}\right)$ as $a \rightarrow \infty$ in the norm resolvent sense, and that $H_{a, L}^{m}$ is reduced by $L^{2}\left(\mathbb{R}^{d N}\right) \otimes \mathcal{F}_{a}$. Put

$$
\begin{aligned}
\mathcal{H} & =L^{2}\left(\mathbb{R}^{d N}\right) \otimes\left[\mathcal{F}_{a} \oplus \mathcal{F}_{a}^{\perp}\right] \\
& =\left[L^{2}\left(\mathbb{R}^{d N}\right) \otimes \mathcal{F}_{a}\right] \oplus\left[L^{2}\left(\mathbb{R}^{d N}\right) \otimes \mathcal{F}_{a}^{\perp}\right]:=\mathcal{H}_{1} \oplus \mathcal{H}_{2} .
\end{aligned}
$$

We see that

$$
\left(\Psi_{2}, H_{a, L}^{m} \Psi_{2}\right) \geq\left(G\left(H_{a, L}^{m}\right)+m\right)\left\|\Psi_{2}\right\|^{2} \quad \text { for } \Psi_{2} \in \mathcal{H}_{2} \cap \mathcal{D}_{m}
$$

and that the dimension of the space generated by vectors $\Psi_{1}$ 's $\in \mathcal{H}_{1} \cap \mathcal{D}_{m}$ so that $\left(\left[H_{a, L}^{m}-m-G\left(H_{a, L}^{m}\right)\right] \Psi_{1}, \Psi_{1}\right) \leq 0$ is finite. Hence we obtain $\left[G\left(H_{a, L}^{m}\right), G\left(H_{a, L}^{m}\right)+\right.$ $m) \cap \sigma\left(H_{a, L}^{m}\right) \subset \sigma_{\mathrm{d}}\left(H_{a, L}^{m}\right)$. Moreover, we prove that $H_{L}^{m}$ converges to $H^{m}$ in the norm resolvent sense as $L \rightarrow \infty$. Hence the norm resolvent convergences of $H_{a, L}^{m}$ to $H_{L}^{m}$, and of $H_{L}^{m}$ to $H^{m}$, imply that

$$
\left[G\left(H^{m}\right), G\left(H^{m}\right)+m\right) \cap \sigma\left(H^{m}\right) \subset \sigma_{\mathrm{d}}\left(H^{m}\right) .
$$

Denote a ground state of $H^{m}$ by $\Omega_{m}$. Taking a subsequence in $m$, we can see that $\Omega_{m}$ weakly converges to vector $\Omega_{g}$. We see that $G\left(H^{m}\right) \rightarrow G(H)$ as $m \rightarrow 0$ and $\Omega_{g} \neq 0$. Thus we conclude that $\Omega_{g}$ is a ground state of $H$ with eigenvalue $G(H)$.

\subsection{Momentum lattice approximation. Define}

$$
\Gamma_{a}:=\left\{k=\left(k_{1}, \ldots, k_{d}\right) \mid k_{\mu}=2 \pi n_{\mu} / a, n_{\mu} \in \mathbb{Z}, \mu=1, \ldots, d\right\} .
$$

We call $k \in \Gamma_{a}$ a lattice point. Let $l_{2}\left(\Gamma_{a}\right)$ be the set of $l_{2}$ sequences over $\Gamma_{a}$. Thus we define $\mathcal{F}_{a}$ as $\mathcal{F}_{a}:=\bigoplus_{n=0}^{\infty} \otimes_{s}^{n}\left[\bigoplus_{r=1}^{d-1} l_{2}\left(\Gamma_{a}\right)\right]$. We identify $l_{2}\left(\Gamma_{a}\right)$ with the subspace of $L^{2}\left(\mathbb{R}^{d}\right)$ consisting of piecewise constant functions on each cube of volume $(2 \pi / a)^{d}$ centered on lattice points. Thus this identification immediately induces the identification of

$$
\underbrace{l_{2}\left(\Gamma_{a}\right) \oplus \ldots \oplus l_{2}\left(\Gamma_{a}\right)}_{d-1}
$$

as a subspace of $W$. Under this identification, we regard $\mathcal{F}_{a}$ as the closed subspace of $\mathcal{F}$. For $m>0$ and $a<\infty$, we define nonnegative self-adjoint operators by

$$
\begin{aligned}
H_{\mathrm{f}, a}^{m} & :=\sum_{r=1}^{d-1} \int\left(\omega_{a}(k)+m\right) a^{\dagger r}(k) a^{r}(k) d k, \\
H_{\mathrm{f}}^{m} & :=\sum_{r=1}^{d-1} \int(\omega(k)+m) a^{\dagger r}(k) a^{r}(k) d k .
\end{aligned}
$$

Here we set $\omega_{a}(k):=\omega\left(k_{a}\right)$, where $k_{a}:=\left(k_{1, a}, \ldots, k_{d, a}\right)$ is the step function

$$
k_{\mu, a}(k):= \begin{cases}2 \pi n / a, & k_{\mu} \in[(2 n-1) \pi / a,(2 n+1) \pi / a), \\ 0, & \text { otherwise. }\end{cases}
$$

Proposition 3.1. $H_{\mathrm{f}, a}^{m}$ and $H_{\mathrm{f}}^{m}$ have the following properties:

(1) $D\left(H_{\mathrm{f}, a}^{m}\right)=D\left(H_{\mathrm{f}}^{m}\right)=D\left(H_{\mathrm{f}}\right) \cap D(N)$ and $H_{\mathrm{f}}^{m}=H_{\mathrm{f}}+m N$. Moreover, $H_{\mathrm{f}, a}^{m}$ has compact resolvent, i.e., $H_{\mathrm{f}, a}^{m}$ has purely discrete spectrum; 
(2) Let $C_{a}:=\sqrt{d}(\pi / a)(1 /(2 m)+1)$. Then, for sufficiently large $a$ and $\Psi \in$ $D\left(H_{\mathrm{f}}^{m}\right)$,

$$
\left\|H_{\mathrm{f}, a}^{m} \Psi\right\| \leq \frac{1+C_{a}}{1-C_{a}}\left\|H_{\mathrm{f}}^{m} \Psi\right\|, \quad\left\|H_{\mathrm{f}}^{m} \Psi\right\| \leq \frac{1+C_{a}}{1-C_{a}}\left\|H_{\mathrm{f}, a}^{m} \Psi\right\| ;
$$

(3) $H_{\mathrm{f}, a}^{m}$ is reduced by $\mathcal{F}_{a}$ with

$$
H_{\mathrm{f}, a}^{m} \Gamma_{\mathcal{F}_{a}}=\left(\frac{a}{2 \pi}\right)^{d} \sum_{r=1}^{d-1} \sum_{k \in \Gamma_{a}}(\omega(k)+m) a^{\dagger r}\left(\chi_{C(k, a)}\right) a^{r}\left(\chi_{C(k, a)}\right),
$$

where $\chi_{C(k, V)}, k \in \Gamma_{a}$, is the characteristic function of

$$
C(k, a):=\left[k_{1}-\frac{\pi}{a}, k_{1}+\frac{\pi}{a}\right) \times \cdots \times\left[k_{d}-\frac{\pi}{a}, k_{d}+\frac{\pi}{a}\right) ;
$$

(4) For $\Psi \in D\left(H_{\mathrm{f}}^{m}\right),\left\|\left(H_{\mathrm{f}}^{m}-H_{\mathrm{f}, a}^{m}\right) \Psi\right\| \leq 2 C_{a} /\left(1-C_{a}\right)\left\|H_{\mathrm{f}}^{m} \Psi\right\|$;

(5) For sufficiently large $a$ and $\Psi \in D\left(H_{\mathrm{f}, a}^{m}\right),\left(\Psi, H_{\mathrm{f}} \Psi\right) \leq\left(\Psi, H_{\mathrm{f}, a}^{m} \Psi\right)$;

(6) Subspace $\mathcal{F}^{\infty} \cap C^{\infty}\left(H_{\mathrm{f}}\right)$ is a core for both $H_{\mathrm{f}}^{m}$ and $H_{\mathrm{f}, a}^{m}$.

Proof. See [11, Lemma 3.1 and Lemma 3.6], 21, p. 367] for (1), (2), (3) and (4). Since, for sufficiently large $a, \omega(k) \leq \omega\left(k_{a}\right)+m,(5)$ follows. Since $H_{\mathrm{f}}$ and $N$ commute, one sees that $\mathcal{F}^{\infty} \cap C^{\infty}\left(H_{\mathrm{f}}\right)$ is an invariant dense subspace for $e^{-t H_{\mathrm{f}}^{m}}, t \geq$ 0 . Hence (6) follows from (2).

We define $A_{a}(x):=\left(A_{1, a}(x), \cdots, A_{d, a}(x)\right)$ by

$$
\begin{aligned}
A_{\mu, a}(x) & =A_{\mu, a}(\hat{\rho}, x) \\
& :=\sum_{r=1}^{d-1} \int d k \sum_{l \in \Gamma_{a}} \chi_{C(l, V)}(k)\left\{\frac{\hat{\rho}(l) e_{\mu}^{r}(l) e^{-i l x}}{\sqrt{2 \omega(l)}} a^{\dagger r}(k)+\frac{\hat{\rho}(-l) e_{\mu}^{r}(l) e^{i l x}}{\sqrt{2 \omega(l)}} a^{r}(k)\right\} .
\end{aligned}
$$

Thus $H_{a, L}^{m}$ is defined by

$$
H_{a, L}^{m}:=H_{\mathrm{el}} \otimes I+I \otimes H_{\mathrm{f}, a}^{m}+e H_{\mathrm{I}, a}+e^{2} H_{\mathrm{II}, a}:=H_{0, a}^{m}+e H_{\mathrm{I}, a}+e^{2} H_{\mathrm{II}, a} .
$$

Here $H_{\mathrm{I}, a}:=H_{\mathrm{I}, a}\left(\hat{\rho}_{L}\right)$ and $H_{\mathrm{II}, a}:=H_{\mathrm{II}, a}\left(\hat{\rho}_{L}\right)$ are defined by $H_{\mathrm{I}}$ and $H_{\mathrm{II}}$ with $A\left(\hat{\rho}, x^{j}\right)$ replaced by $A_{a}\left(\hat{\rho}_{L}, x^{j}\right)$, respectively. We also define

$$
\begin{gathered}
H^{m}:=H^{m}(\hat{\rho}):=H_{\mathrm{el}} \otimes I+I \otimes H_{\mathrm{f}}^{m}+e H_{\mathrm{I}}+e^{2} H_{\mathrm{II}}:=H_{0}^{m}+e H_{\mathrm{I}}+e^{2} H_{\mathrm{II}}, \\
H_{L}^{m}:=H^{m}\left(\hat{\rho}_{L}\right) .
\end{gathered}
$$

Note that $\lim _{a \rightarrow \infty}\left(1+C_{a}\right) /\left(1-C_{a}\right)=1$. Proposition 3.1 (1), (2), and the commutativity of $H_{\mathrm{f}}$ and $N^{1 / 2}$ yield that, for $\Psi \in D\left(H_{\mathrm{f}}\right) \cap D(N)$ and sufficiently large $a$,

$$
\left\|H_{\mathrm{f}} \Psi\right\| \leq\left\|H_{\mathrm{f}}^{m} \Psi\right\| \leq 2\left\|H_{\mathrm{f}, a}^{m} \Psi\right\| .
$$

Lemma 3.2. Let a be sufficiently large. Then there exist constants $k_{1}$ and $k_{2}$ which are independent of a so that

$$
\begin{aligned}
\left\|H_{\mathrm{I}, a} \Psi\right\| & \leq k_{1}\left(\left\|H_{0} \Psi\right\|+\|\Psi\|\right), \\
\left\|H_{\mathrm{II}, a} \Psi\right\| & \leq k_{2}\left(\left\|H_{0} \Psi\right\|+\|\Psi\|\right) .
\end{aligned}
$$


Proof. From (2.13), it follows that $\left\|H_{\mathrm{I}, a} \Psi\right\| \leq \widetilde{M}_{\mathrm{I}}(a)\left(\left\|H_{0} \Psi\right\|+\|\Psi\|\right)$, where $\widetilde{M}_{\mathrm{I}}(a)$ is defined by $M_{\mathrm{I}}$ with $\left\|\hat{\rho}_{L}\right\|_{-2}$ and $\left\|\hat{\rho}_{L}\right\|_{-1}$ replaced by $\left\|\sum_{l \in \Gamma_{a}} \hat{\rho}_{L}(l) \chi_{C(l, V)} / \sqrt{\omega(l)}\right\|_{-1}$ and $\left\|\sum_{l \in \Gamma_{a}} \hat{\rho}_{L}(l) \chi_{C(l, V)} / \sqrt{\omega(l)}\right\|_{0}$, respectively. Since, by the compactness and the continuity of $\hat{\rho}_{L} / \sqrt{\omega}$, the Lebesgue dominated convergence theorem yields that

$$
\left\|\sum_{l \in \Gamma_{a}} \hat{\rho}_{L}(l) \chi_{C(l, V)} / \sqrt{\omega(l)}\right\|_{n} \longrightarrow\left\|\hat{\rho}_{L} / \sqrt{\omega}\right\|_{n}
$$

as $a \rightarrow \infty$ for $n=-1,0$, one can choose a constant $k_{1}$ so that $\widetilde{M}_{\mathrm{I}}(a)<k_{1}$ for sufficiently large $a$. Thus (3.3) follows. (3.4) is similarly proven.

Proposition 3.3. (1) Assume that a is sufficiently large. Then $H_{a, L}^{m}$ is self-adjoint on $\mathcal{D}_{m}:=D\left(H_{\mathrm{el}} \otimes I\right) \cap D\left(I \otimes H_{\mathrm{f}}^{m}\right)$, bounded below, and essentially self-adjoint on any core for $H_{0, a}^{m}$. (2) $H^{m}$ is self-adjoint on $\mathcal{D}_{m}$, bounded below, and essentially self-adjoint on any core for $H_{0}^{m}$.

Proof. By (3.2) we see that, for sufficiently large $a$,

$$
\left\|H_{0} \Psi\right\| \leq\left\|H_{0}^{m} \Psi\right\|+2|\mathbf{g}|\|\Psi\| \leq 2\left\|H_{0, a}^{m} \Psi\right\|+5|\mathbf{g}|\|\Psi\| .
$$

Due to (3.6) and Lemma [3.2, we see that, with $k_{1}$ and $k_{2}$ in (3.3) and (3.4), $\left\|H_{\mathrm{I}, a} \Psi\right\| \leq k_{1}\left(2\left\|H_{0, a}^{m} \Psi\right\|+(5|\mathbf{g}|+1)\|\Psi\|\right)$ and $\left\|H_{\mathrm{II}, a} \Psi\right\| \leq k_{2}\left(2\left\|H_{0, a}^{m} \Psi\right\|+(5|\mathbf{g}|+\right.$ $1)\|\Psi\|)$. Hence the Kato-Rellich theorem yields (1). Also by (2.13) and (3.6), we see that

$$
\begin{aligned}
\left\|H_{\mathrm{I}} \Psi\right\| & \leq M_{\mathrm{I}}\left(\left\|H_{0}^{m} \Psi\right\|+(2|\mathbf{g}|+1)\|\Psi\|\right), \\
\left\|H_{\mathrm{II}} \Psi\right\| & \leq M_{\mathrm{II}}\left(\left\|H_{0}^{m} \Psi\right\|+(2|\mathbf{g}|+1)\|\Psi\|\right) .
\end{aligned}
$$

Hence again the Kato-Rellich theorem yields (2).

Lemma 3.4. There exist constants $c_{1}, c_{2}$ and $c_{3}$ so that, for $\Psi \in \mathcal{D}_{m}$

$$
\begin{aligned}
& \left\|H_{0} \Psi\right\| \leq c_{1}\left(\left\|H_{a, L}^{m} \Psi\right\|+\|\Psi\|\right), \\
& \left\|H_{0} \Psi\right\| \leq c_{2}\left(\left\|H^{m} \Psi\right\|+\|\Psi\|\right), \\
& \left\|H_{0}^{m} \Psi\right\| \leq c_{3}\left(\left\|H^{m} \Psi\right\|+\|\Psi\|\right),
\end{aligned}
$$

where $c_{1}$ is independent of sufficiently large $a$.

Proof. By (3.3), (3.4) and (3.6), we see that

$$
\begin{aligned}
\left\|H_{0} \Psi\right\| \leq & \left\{2\left\|H_{a, L}^{m} \Psi\right\|\right. \\
& \left.+\left(5|\mathbf{g}|+\left(2 e k_{1}+2 e^{2} k_{2}\right)(5|\mathbf{g}|+1)\right)\|\Psi\|\right\} /\left(1-2 e k_{1}-2 e^{2} k_{2}\right) .
\end{aligned}
$$

Thus (3.8) follows. Similarly we see that, by (2.13) and (3.6),

$$
\left\|H_{0} \Psi\right\| \leq\left\{\left\|H^{m} \Psi\right\|+\left(2|\mathbf{g}|+\left(e M_{\mathrm{I}}+e^{2} M_{\mathrm{II}}\right)(2|\mathbf{g}|+1)\right)\|\Psi\|\right\} /\left(1-e M_{\mathrm{I}}-e^{2} M_{\mathrm{II}}\right),
$$

and by 3.7 and 3.6),

$$
\left\|H_{0}^{m} \Psi\right\| \leq\left\{\left\|H^{m} \Psi\right\|+\left(e M_{\mathrm{I}}+e^{2} M_{\mathrm{II}}\right)(2|\mathbf{g}|+1)\|\Psi\|\right\} /\left(1-e M_{\mathrm{I}}-e^{2} M_{\mathrm{II}}\right),
$$

which employ (3.9) and (3.10).

The following lemma is a key lemma in this section.

Lemma 3.5. Let us denote the uniform norm of bounded operators on $\mathcal{H}$ by $\|\cdot\|$. Then, for all $z \in \mathbb{C} \backslash \mathbb{R}$, we have $\lim _{a \rightarrow \infty}\left\|\left(H_{a, L}^{m}-z\right)^{-1}-\left(H_{L}^{m}-z\right)^{-1}\right\|=0$. 
Proof. Simply, we put $H_{a, L}^{m}=H_{a}, H_{L}^{m}=H, A_{\mu}\left(\hat{\rho}_{L}, x^{j}\right)=A_{\mu}$ and $A_{\mu, a}\left(\hat{\rho}_{L}, x^{j}\right)=$ $A_{\mu, a}$; moreover, we abbreviate $I \otimes X$ and $X \otimes I$ by $X$ in this proof. Noting that $\left|e^{-i k x}-e^{-i l x}\right| \leq|k-l||x|$ for $k, l, x \in \mathbb{R}^{d}$, we see that

$$
\left\|\frac{\hat{\rho}_{L} e_{\mu}^{r} e^{-i k x^{j}}}{\sqrt{2 \omega}}-\sum_{l \in \Gamma_{a}} \frac{\hat{\rho}_{L}(l) e_{\mu}^{r}(l) e^{-i l x^{j}}}{\sqrt{2 \omega(l)}} \chi_{C(l, a)}\right\|_{n} \leq S_{\mu r n}+\left|x^{j}\right| T_{\mu r n}
$$

where

$$
\begin{aligned}
S_{\mu r n} & :=\left\|\frac{\hat{\rho}_{L} e_{\mu}^{r}}{\sqrt{2 \omega}}-\sum_{l \in \Gamma_{a}} \frac{\hat{\rho}_{L}(l) e_{\mu}^{r}(l)}{\sqrt{2 \omega(l)}} \chi_{C(l, a)}\right\|_{-n}, \\
T_{\mu r n} & :=\left\|\sum_{l \in \Gamma_{a}} \frac{\hat{\rho}_{L}(l) e_{\mu}^{r}(l)}{\sqrt{2 \omega(l)}} \chi_{C(l, a)} \mid \cdot-l\right\| \|_{-n} .
\end{aligned}
$$

Since $\hat{\rho}_{L} e_{\mu}^{r}$ has a compact support, we have $\lim _{a \rightarrow 0} S_{\mu r n}=0$ and $\lim _{a \rightarrow 0} T_{\mu r n}=0$ for $n=1,0$. Let

$$
S_{n}:=\max _{r=1, . ., d-1, \mu=1, . ., d} S_{\mu r n}
$$

and

$$
T_{n}:=\max _{r=1, . ., d-1, \mu=1, . ., d} T_{\mu r n} .
$$

We have

$$
(H-z)^{-1}-\left(H_{a}-z\right)^{-1}=e \mathrm{I}(a)+e^{2} \operatorname{II}(a)+\operatorname{III}(a),
$$

where

$$
\begin{aligned}
& \mathrm{I}(a):=-\sum_{j=1}^{N} \sum_{\mu=1}^{d}\left(H_{a}-z\right)^{-1}\left(A_{\mu}-A_{\mu, a}\right) p_{\mu}^{j}(H-z)^{-1}, \\
& \operatorname{II}(a):=\frac{1}{2} \sum_{j=1}^{N} \sum_{\mu=1}^{d}\left(H_{a}-z\right)\left(A_{\mu}^{2}-A_{\mu, a}^{2}\right)(H-z)^{-1}, \\
& \operatorname{III}(a):=\left(H_{a}-z\right)^{-1}\left(H_{\mathrm{f}}^{m}-H_{\mathrm{f}, a}^{m}\right)(H-z)^{-1} .
\end{aligned}
$$

We shall show that $\mathrm{I}(a), \operatorname{II}(a)$ and $\operatorname{III}(a)$ converge to zero uniformly as $a \rightarrow 0$, respectively. By (3.8), (3.9) and (3.10), we can see that there exist positive constants $\mathbf{A}, \mathbf{B}$ and $\mathbf{C}$ so that

$$
\begin{aligned}
& \max \left\{\left\|\left(H_{0}-\bar{z}\right)\left(H_{a}-\bar{z}\right)^{-1}\right\|,\left\|\left(H_{0}-z\right)\left(H_{a}-z\right)^{-1}\right\|\right\} \leq \mathbf{A}, \\
& \max \left\{\left\|\left(H_{0}-\bar{z}\right)(H-\bar{z})^{-1}\right\|,\left\|\left(H_{0}-z\right)(H-z)^{-1}\right\|\right\} \leq \mathbf{B}, \\
& \max \left\{\left\|\left(H_{0}^{m}-\bar{z}\right)(H-\bar{z})^{-1}\right\|,\left\|\left(H_{0}^{m}-z\right)(H-z)^{-1}\right\|\right\} \leq \mathbf{C},
\end{aligned}
$$

where $\mathbf{A}$ is independent of sufficiently large $a$. Moreover, by (2.28), (2.21), (2.22) and (2.23),

$$
\begin{gathered}
\left(\left|x^{j}\right| \otimes H_{\mathrm{f}}^{1 / 2}\right)\left(H_{0}-\bar{z}\right)^{-1},\left|x^{j}\right|\left(H_{0}-\bar{z}\right)^{-1}, \\
p_{\mu}^{j}\left(H_{0}-z\right)^{-1}, H_{\mathrm{f}}^{1 / 2}\left(H_{0}-\bar{z}\right)
\end{gathered}
$$


are bounded. Let $V_{n}:=S_{n}+\left|x^{j}\right| T_{n}$. We see that, for $\Psi, \Phi \in \mathcal{H}$,

$$
\begin{aligned}
& |(\Psi, \mathrm{I}(a) \Phi)| \leq \sum_{j=1}^{N} \sum_{\mu=1}^{d}\left|\left(\left(A_{\mu}-A_{\mu, a}\right)\left(H_{a}-\bar{z}\right)^{-1} \Psi, p_{\mu}^{j}(H-z)^{-1} \Phi\right)\right| \\
& \leq \sum_{j=1}^{N} \sum_{\mu=1}^{d}(d-1)\left\|p_{\mu}^{j}(H-z)^{-1}\right\|\left\{\left\|2 V_{1} H_{\mathrm{f}}^{1 / 2}\left(H_{a}-\bar{z}\right)^{-1}\right\|\right. \\
& \left.+\left\|V_{0}\left(H_{a}-\bar{z}\right)^{-1}\right\|\right\}\|\Psi\|\|\Phi\| \\
& \leq \sum_{j=1}^{N} \sum_{\mu=1}^{d}(d-1)\left\{\left\|2 V_{1} H_{\mathrm{f}}^{1 / 2}\left(H_{0}-\bar{z}\right)^{-1}\left(H_{0}-\bar{z}\right)\left(H_{a}-\bar{z}\right)^{-1}\right\|\right. \\
& \left.+\left\|V_{0}\left(H_{0}-\bar{z}\right)^{-1}\left(H_{0}-\bar{z}\right)\left(H_{a}-\bar{z}\right)^{-1}\right\|\right\} \\
& \times\left\|p_{\mu}^{j}\left(H_{0}-z\right)^{-1}\left(H_{0}-z\right)(H-z)^{-1}\right\|\|\Psi\|\|\| \Phi \| \\
& \leq \sum_{j=1}^{N} \sum_{\mu=1}^{d}(d-1) \mathbf{A B}\left\{2 S_{1}\left\|H_{\mathrm{f}}^{1 / 2}\left(H_{0}-\bar{z}\right)^{-1}\right\| S_{0}\left\|\left(H_{0}-\bar{z}\right)^{-1}\right\|\right. \\
& \left.+2 T_{1}\left\|\left(\left|x^{j}\right| \otimes H_{\mathrm{f}}^{1 / 2}\right)\left(H_{0}-\bar{z}\right)^{-1}\right\|+T_{0}\left\|\left|x^{j}\right|\left(H_{0}-\bar{z}\right)^{-1}\right\|\right\} \\
& \times\left\|p_{\mu}^{j}\left(H_{0}-z\right)^{-1}\right\|\|\Psi\|\|\Phi\| \text {. }
\end{aligned}
$$

Here the second inequality above is due to (2.6). Let

$$
\alpha:=\max \left\{\begin{array}{l}
\left\|\left(\left|x^{j}\right| \otimes H_{\mathrm{f}}^{1 / 2}\right)\left(H_{0}-\bar{z}\right)^{-1}\right\|,\left\|\left|x^{j}\right|\left(H_{0}-\bar{z}\right)^{-1}\right\| \\
\left\|p_{\mu}^{j}\left(H_{0}-z\right)^{-1}\right\|,\left\|H_{\mathrm{f}}^{1 / 2}\left(H_{0}-\bar{z}\right)\right\|,\left\|\left(H_{0}-\bar{z}\right)^{-1}\right\|
\end{array}\right\}
$$

Let $U(a):=2 S_{1}+S_{0}+2 T_{1}+T_{0}$. We have

$$
|(\Psi, \mathrm{I}(a) \Phi)| \leq d(d-1) N \alpha^{2} \mathbf{A B} U(a)\|\Psi\|\|\Phi\|
$$

Thus $\|\mathrm{I}(a)\| \leq d(d-1) N \alpha^{2} \mathbf{A B} U(a)$, which implies that

$$
\lim _{a \rightarrow \infty}\|\mathrm{I}(a)\|=0
$$

Next, we see that

$$
\begin{gathered}
\operatorname{II}(a)=\frac{1}{2} \sum_{j=1}^{N} \sum_{\mu=1}^{d} \mathcal{M}_{j, \mu}+\frac{1}{2} \sum_{j=1}^{N} \sum_{\mu=1}^{d} \mathcal{N}_{j, \mu}, \\
\mathcal{M}_{j, \mu}:=\left(H_{a}-z\right)^{-1}\left(A_{\mu}-A_{\mu, a}\right) A_{\mu}(H-z)^{-1}, \\
\mathcal{N}_{j, \mu}:=\left(H_{a}-z\right)^{-1} A_{\mu, a}\left(A_{\mu}-A_{\mu, a}\right)(H-z)^{-1} .
\end{gathered}
$$

In addition to (3.12), $H_{\mathrm{f}}^{1 / 2}\left(H_{0}-z\right)^{-1}$ and $\left|x^{j}\right|\left(H_{0}-z\right)^{-1}$ are also bounded by (2.23) and (2.22). Using (2.6), we have 


$$
\begin{aligned}
& \left|\left(\Psi, \mathcal{M}_{j, \mu} \Phi\right)\right|=\left|\left(\left(A_{\mu}-A_{\mu, a}\right)\left(H_{a}-\bar{z}\right)^{-1} \Psi, A_{\mu}(H-z)^{-1} \Phi\right)\right| \\
& \leq(d-1)\left\{\left\|2 V_{1} H_{\mathrm{f}}^{1 / 2}\left(H_{a}-\bar{z}\right)^{-1}\right\|+\left\|V_{0}\left(H_{a}-\bar{z}\right)^{-1}\right\|\right\} \\
& \quad \times \frac{1}{\sqrt{2}}\left\{2\left\|\hat{\rho}_{L}\right\|_{-2}\left\|H_{\mathrm{f}}^{1 / 2}(H-z)^{-1}\right\|+\left\|\hat{\rho}_{L}\right\|_{-1}\left\|(H-z)^{-1}\right\|\right\}\|\Psi\|\|\Phi\| \\
& =\left\{\left\|2 V_{1} H_{\mathrm{f}}^{1 / 2}\left(H_{0}-\bar{z}\right)^{-1}\left(H_{0}-\bar{z}\right)\left(H_{a}-\bar{z}\right)^{-1}\right\|\right. \\
& \left.\quad+\left\|V_{0}\left(H_{0}-\bar{z}\right)^{-1}\left(H_{0}-\bar{z}\right)\left(H_{a}-\bar{z}\right)^{-1}\right\|\right\} \\
& \quad \times \frac{d-1}{\sqrt{2}}\left\{2\left\|\hat{\rho}_{L}\right\|_{-2}\left\|H_{\mathrm{f}}^{1 / 2}\left(H_{0}-z\right)^{-1}\left(H_{0}-z\right)(H-z)^{-1}\right\|\right. \\
& \left.\quad+\left\|\hat{\rho}_{L}\right\|_{-1}\left\|\left(H_{0}-z\right)^{-1}\left(H_{0}-z\right)(H-z)^{-1}\right\|\right\}\|\Psi\|\|\Phi\| \\
& \leq(d-1) \mathbf{A B}\left\{2 S_{1}\left\|H_{\mathrm{f}}^{1 / 2}\left(H_{0}-\bar{z}\right)^{-1}\right\|+2 T_{1}\left\|\left(\left|x^{j}\right| \otimes H_{\mathrm{f}}^{1 / 2}\right)\left(H_{0}-\bar{z}\right)^{-1}\right\|\right. \\
& \left.\quad+S_{0}\left\|\left(H_{0}-\bar{z}\right)^{-1}\right\|+T_{0}\left\|\left|x^{j}\right|\left(H_{0}-\bar{z}\right)^{-1}\right\|\right\} \\
& \quad \times \frac{1}{\sqrt{2}}\left\{2\left\|\hat{\rho}_{L}\right\|_{-2}\left\|H_{\mathrm{f}}^{1 / 2}\left(H_{0}-z\right)^{-1}\right\|+\left\|\hat{\rho}_{L}\right\|\left\|_{-1}\right\|\left(H_{0}-z\right)^{-1} \|\right\}\|\Psi\|\|\Phi\| .
\end{aligned}
$$

Let $\beta:=\max \left\{\alpha,\left\|H_{\mathrm{f}}^{1 / 2}\left(H_{0}-z\right)^{-1}\right\|,\left\|\left|x^{j}\right|\left(H_{0}-z\right)^{-1}\right\|,\left\|\left(H_{0}-z\right)^{-1}\right\|\right\}$. Then

$$
\left|\left(\Psi, \mathcal{M}_{j, \mu} \Phi\right)\right| \leq \frac{d-1}{\sqrt{2}} \mathbf{A B} \beta^{2} U(a)\left(2\left\|\hat{\rho}_{L}\right\|_{-2}+\left\|\hat{\rho}_{L}\right\|_{-1}\right)\|\Psi\|\|\Phi\| .
$$

Hence $\left\|\mathcal{M}_{j, \mu}\right\| \leq \mathbf{A B} \beta^{2}(d-1) U(a)\left(2\left\|\hat{\rho}_{L}\right\|_{-2}+\left\|\hat{\rho}_{L}\right\|_{-1}\right) / \sqrt{2}$. Thus $\lim _{a \rightarrow \infty}\left\|\mathcal{M}_{j, \mu}\right\|$ $=0$ for $j=1, \ldots, N, \mu=1, \ldots, d$. Similarly one can show that $\left\|\mathcal{N}_{j, \mu}\right\|$ also goes to zero as $a \rightarrow 0$ for $j=1, \ldots, N, \mu=1, \ldots, d$. Thus we conclude that

$$
\lim _{a \rightarrow \infty}\|\mathrm{II}(a)\|=0 \text {. }
$$

Next since $\left\|H_{\mathrm{f}}^{m} \Psi\right\| \leq\left\|H_{0}^{m} \Psi\right\|+2|\mathbf{g}|\|\Psi\|, H_{\mathrm{f}}^{m}\left(H_{0}^{m}-z\right)^{-1}$ is bounded on $\mathcal{H}$. Due to Proposition 3.1 (4) and $\left\|\left(H_{a}-z\right)^{-1}\right\| \leq 1 /|\Im z|$, it follows that

$$
\|\operatorname{III}(a)\| \leq \frac{C}{|\Im z|} \frac{2 C_{a}}{1-C_{a}}\left\|H_{\mathrm{f}}^{m}\left(H_{0}^{m}-z\right)^{-1}\right\| .
$$

Then $\lim _{a \rightarrow 0} C_{a} /\left(1-C_{a}\right)=0$ implies that

$$
\lim _{a \rightarrow \infty}\|\operatorname{III}(a)\|=0 .
$$

Hence we get the desired results.

Lemma 3.6. For all $z \in \mathbb{C} \backslash \mathbb{R}$, we have $\lim _{L \rightarrow \infty}\left\|\left(H_{L}^{m}-z\right)^{-1}-\left(H^{m}-z\right)^{-1}\right\|=0$.

Proof. Noting that $\left\|\hat{\rho}_{L}-\hat{\rho}\right\|_{n} \rightarrow 0$ as $L \rightarrow \infty$ for $n=-2,-1$, one can prove the lemma easily. Details are omitted.

Let $\mathcal{H}_{1}$ and $\mathcal{H}_{2}$ be the closed subspaces in (3.1).

Lemma 3.7. Assume that a is sufficiently large. Then $H_{a, L}^{m}$ is reduced by $\mathcal{H}_{1}$. Moreover,

$$
\left(\Psi_{2}, H_{a, L}^{m} \Psi_{2}\right) \geq\left(G\left(H_{a, L}^{m}\right)+m\right)\left\|\Psi_{2}\right\|^{2}, \quad \Psi_{2} \in \mathcal{H}_{2} \cap \mathcal{D}_{m} .
$$

Proof. We write the projection of $L^{2}\left(\mathbb{R}^{d}\right)$ to $l_{2}\left(\Gamma_{a}\right)$ as $p_{a}$. Then

$$
\tilde{p}_{a}=\underbrace{p_{a} \oplus \ldots \oplus p_{a}}_{d-1}
$$


becomes the projection of $W$ to

$$
\underbrace{l_{2}\left(\Gamma_{a}\right) \oplus \ldots \oplus l_{2}\left(\Gamma_{a}\right)}_{d-1} .
$$

Concretely $p_{a}$ reacts to $f \in C_{0}^{\infty}\left(\mathbb{R}^{d}\right)$ as $p_{a} f(\cdot)=\sum_{l \in \Gamma_{a}} \chi_{C(l, a)}(\cdot) f(l)$. Operator $\tilde{P}_{a}$ is defined by $\tilde{P}_{a} a^{\dagger}\left(F_{1}\right) \ldots a^{\dagger}\left(F_{n}\right) \Omega:=a^{\dagger}\left(\tilde{p}_{a} F_{1}\right) \ldots a^{\dagger}\left(\tilde{p}_{a} F_{n}\right) \Omega$, for $F_{1}, \ldots, F_{n} \in W$, and $\tilde{P}_{a} \Omega_{\mathrm{f}}:=\Omega$, and we extend it on $\mathcal{F}$ linearly. The projection of $\mathcal{H}$ to $\mathcal{H}_{1}$ is eventually, under identification (2.10), given by $P_{a}=\int_{\mathbb{R}^{d N}}^{\oplus} \tilde{P}_{a} d x$. Taking vectors of the form $\Psi=u \otimes a^{\dagger r_{1}}\left(f_{1}\right) \ldots a^{\dagger r_{n}}\left(f_{n}\right) \Omega, u, f_{1}, \ldots, f_{n} \in C_{0}^{\infty}\left(\mathbb{R}^{d}\right)$, we can see, from the definition of $A_{a}\left(x^{j}\right)$, that $H_{\mathrm{I}, a} P_{a} \Psi=P_{a} H_{\mathrm{I}, a} \Psi$ and $H_{\mathrm{II}, a} P_{a} \Psi=P_{a} H_{\mathrm{II}, a} \Psi$. From Proposition 3.1 (2) it follows that $H_{0, a}^{m} P_{a} \Psi=P_{a} H_{0, a}^{m} \Psi$. Thus we have

$$
H_{a, L}^{m} P_{a} \Psi=P_{a} H_{a, L}^{m} \Psi .
$$

Since the set of the finite linear sums of $\Psi$ 's is a core for $H_{a, L}^{m}$ by Proposition 3.3 (1), (3.14) extends to $\Psi \in D\left(H_{a, L}^{m}\right)$. Hence it follows that $H_{a, L}^{m}$ is reduced by $\mathcal{H}_{1}$. (3.13) is proven quite similarly as in [11, 21]. Hence it is omitted.

For self-adjoint operator $T$, we write the associated spectral projection onto Borel set $\mathcal{B} \subset \mathbb{R}$ as $E_{T}(\mathcal{B})$, and the range of $E_{T}(\mathcal{B})$ as $\operatorname{Ran} E_{T}(\mathcal{B})$, and the dimension of the range of $E_{T}(\mathcal{B})$ as $\operatorname{dim} E_{T}(\mathcal{B})$.

Lemma 3.8. Let a be sufficiently large. Then

$$
\operatorname{dim} E_{H_{a, L}^{m}}\left(\left[G\left(H_{a, L}^{m}\right), G\left(H_{a, L}^{m}\right)+m\right)\right) \Gamma_{\mathcal{H}_{1}}<\infty .
$$

Proof. From Proposition [3.1 (5), for sufficiently large $a$, we see that

$$
\left(\Psi, H_{0} \Psi\right) \leq\left(\Psi, H_{0, a}^{m} \Psi\right), \quad \Psi \in \mathcal{D}_{m} .
$$

From 2.14 and (3.5), there exist $\eta_{1}, \eta_{2}, \eta_{3}$ and $\eta_{4}$, which are independent of sufficiently large $a$, so that $\left|\left(\Psi, H_{\mathrm{I}, a} \Psi\right)\right| \leq \eta_{1}\left(\Psi, H_{0} \Psi\right)+\eta_{2}\|\Psi\|$ and $\left|\left(\Psi, H_{\mathrm{II}, a} \Psi\right)\right| \leq$ $\eta_{3}\left(\Psi, H_{0} \Psi\right)+\eta_{4}\|\Psi\|$. Combining this and (3.15), we see that $\left|\left(\Psi, H_{\mathrm{I}, a} \Psi\right)\right| \leq$ $\eta_{1}\left(\Psi, H_{0, a}^{m} \Psi\right)+\eta_{2}\|\Psi\|^{2}$ and $\left|\left(\Psi, H_{\mathrm{II}, a} \Psi\right)\right| \leq \eta_{3}\left(\Psi, H_{0, a}^{m} \Psi\right)+\eta_{4}\|\Psi\|^{2}$. Then

$$
\left(\Psi, H_{a, L}^{m} \Psi\right) \geq\left(1-e \eta_{1}-e^{2} \eta_{3}\right)\left(\Psi, H_{0, a}^{m} \Psi\right)-\left(e \eta_{2}+e^{2} \eta_{4}\right)\|\Psi\|^{2} .
$$

Put $\epsilon:=1-e \eta_{1}-e^{2} \eta_{3}, \tilde{\epsilon}:=e \eta_{2}+e^{2} \eta_{4}$ and $H_{a}:=H_{a, L}^{m}$. Define $\overline{H_{\mathrm{el}}}:=H_{\mathrm{el}}-\mathbf{g} \geq 0$. By $\left(f, \overline{H_{\mathrm{el}}} f\right) \geq\left(f,(\Sigma-\mathbf{g}) E_{\overline{H_{\mathrm{el}}}}([\Sigma-\mathbf{g}, \infty)) f\right)$, for $f \in D\left(H_{\mathrm{el}}\right)$ and $\Sigma>\mathbf{g}$, we see that by (3.16),

$$
\begin{aligned}
&(\left.\left.H_{a}-m-G\left(H_{a}\right)\right) \Psi_{1}, \Psi_{1}\right) \\
& \geq\left(\left(\epsilon H_{0, a}^{m}-\epsilon \mathbf{g}\right) \Psi_{1}-\left(\tilde{\epsilon}+m+G\left(H_{a}\right)-\epsilon \mathbf{g}\right) \Psi_{1}, \Psi_{1}\right) \\
& \geq\left(\left\{E_{\overline{H_{\mathrm{el}}}}([0, \Sigma-\mathbf{g}))+E_{\overline{H_{\mathrm{el}}}}([\Sigma-\mathbf{g}, \infty))\right\}\right. \\
&\left.\otimes\left\{\epsilon H_{\mathrm{f}, a}^{m}-\left(\tilde{\epsilon}+m+G\left(H_{a}\right)-\epsilon \mathbf{g}\right)\right\} \Psi_{1}, \Psi_{1}\right) \\
&+\left(\epsilon(\Sigma-\mathbf{g}) E_{\overline{H_{\mathrm{el}}}}([\Sigma-\mathbf{g}, \infty)) \otimes I \Psi_{1}, \Psi_{1}\right) \\
& \geq\left(E_{\overline{H_{\mathrm{el}}}}([0, \Sigma-\mathbf{g})) \otimes\left[\epsilon H_{\mathrm{f}, a}^{m}-\left(\tilde{\epsilon}+m+G\left(H_{a}\right)-\epsilon \mathbf{g}\right)\right] \Psi_{1}, \Psi_{1}\right) \\
& \quad+\left(\epsilon(\Sigma-\mathbf{g})-\left(\tilde{\epsilon}+m+G\left(H_{a}\right)-\epsilon \mathbf{g}\right)\right)\left(E_{\overline{H_{\mathrm{el}}}}([\Sigma-\mathbf{g}, \infty)) \otimes I \Psi_{1}, \Psi_{1}\right) \\
& \quad+\left(E_{\overline{H_{\mathrm{el}}}}([\Sigma-\mathbf{g}, \infty)) \otimes \epsilon H_{\mathrm{f}, a}^{m} \Psi_{1}, \Psi_{1}\right) .
\end{aligned}
$$


Taking $\Sigma$ sufficiently large, we see that (3.17) is nonnegative. It is clear that (3.18) is nonnegative. Thus, taking a sufficiently large, we see that

$$
\begin{aligned}
& \left(\left(H_{a}-m-G\left(H_{a}\right)\right) \Psi_{1}, \Psi_{1}\right) \\
& \quad \geq\left(E_{\overline{H_{\mathrm{el}}}}([0, \Sigma-\mathbf{g})) \otimes\left[\epsilon H_{\mathrm{f}, a}^{m}-\left(\tilde{\epsilon}+m+G\left(H_{a}\right)-\epsilon \mathbf{g}\right)\right] \Psi_{1}, \Psi_{1}\right),
\end{aligned}
$$

which implies that

$$
\begin{gathered}
\operatorname{Ran}_{H_{a}}\left(\left[G\left(H_{a}\right), G\left(H_{a}\right)+m\right)\right)\left\lceil\mathcal{H}_{1}\right. \\
\subset \operatorname{Ran}\left\{E_{\overline{H_{\mathrm{el}}}}([0, \Sigma-\mathbf{g})) \otimes E_{H_{\mathrm{f}, a}^{m}}\left(\left[0, \frac{\tilde{\epsilon}+m+G\left(H_{a}\right)-\epsilon \mathbf{g}}{\epsilon}\right)\right)\left\lceil\mathcal{H}_{1}\right\} .\right.
\end{gathered}
$$

Then, we have

$$
\begin{aligned}
& \operatorname{dim} E_{H_{a}}\left(\left[G\left(H_{a}\right), G\left(H_{a}\right)+m\right)\right)\left\lceil\mathcal{H}_{1}\right. \\
& \leq \operatorname{dim} E_{\overline{H_{\mathrm{el}}}}([0, \Sigma-\mathbf{g})) \times \operatorname{dim} E_{H_{\mathrm{f}, a}^{m}}\left(\left[0, \frac{\tilde{\epsilon}+m+G\left(H_{a}\right)-\epsilon \mathbf{g}}{\epsilon}\right)\right) .
\end{aligned}
$$

Note that $\tilde{\epsilon}+G\left(H_{a}\right)-\epsilon \mathbf{g} \geq 0$. By Hypothesis 3 and Proposition [3.1 (1), the right-hand side of (3.19) is finite. Thus the desired results follow.

We show a general lemma.

Lemma 3.9 ([38, Lemma 4.6]). Let both of $T_{n}$ and $T$ be self-adjoint on a Hilbert space and bounded below. Suppose that $T_{n} \rightarrow T$ as $n \rightarrow \infty$ in the norm resolvent sense, and that $T_{n}$ has purely discrete spectrum in $\left[G\left(T_{n}\right), G\left(T_{n}\right)+C\right.$ ) with some $C>0$. Then $\sigma(T) \cap[G(T), G(T)+C) \subset \sigma_{\mathrm{d}}(T)$.

Lemma 3.10. We have $\sigma\left(H^{m}\right) \cap\left[G\left(H^{m}\right), G\left(H^{m}\right)+m\right) \subset \sigma_{\mathrm{d}}\left(H^{m}\right)$. In particular, the ground states of $H^{m}$ exist.

Proof. From (3.13) it follows that $\operatorname{Ran} E_{H_{a, L}^{m}}\left(\left[G\left(H_{a, L}^{m}\right), G\left(H_{a, L}^{m}\right)+m\right)\right) \Gamma_{\mathcal{H}_{2}}=\{0\}$. Then we have for sufficiently large $a$,

$$
\begin{aligned}
& \operatorname{Ran} E_{H_{a, L}^{m}}\left(\left[G\left(H_{a, L}^{m}\right), G\left(H_{a, L}^{m}\right)+m\right)\right) \\
= & \operatorname{Ran} E_{H_{a, L}^{m}}\left(\left[G\left(H_{a, L}^{m}\right), G\left(H_{a, L}^{m}\right)+m\right)\right)\left\lceil\mathcal{H}_{1}\right. \\
& \oplus \operatorname{Ran} E_{H_{a, L}^{m}}\left(\left[G\left(H_{a, L}^{m}\right), G\left(H_{a, L}^{m}\right)+m\right)\right)\left\lceil\mathcal{H}_{2}\right. \\
= & \operatorname{Ran} E_{H_{a, L}^{m}}\left(\left[G\left(H_{a, L}^{m}\right), G\left(H_{a, L}^{m}\right)+m\right)\right)\left\lceil\mathcal{H}_{1}\right.
\end{aligned}
$$

Then from Lemma 3.8 it follows that

$\operatorname{dim} E_{H_{a, L}^{m}}\left(\left[G\left(H_{a, L}^{m}\right), G\left(H_{a, L}^{m}\right)+m\right)\right)=\operatorname{dim} E_{H_{a, L}^{m}}\left(\left[G\left(H_{a, L}^{m}\right), G\left(H_{a, L}^{m}\right)+m\right)\right)\left\lceil\mathcal{H}_{1}<\infty\right.$,

which implies that $H_{a, L}^{m}$ has purely discrete spectrum in $\left.\left[G\left(H_{a, L}^{m}\right), G\left(H_{a, L}^{m}\right)+m\right)\right)$. By Lemmas 3.5 and 3.9, $H_{L}^{m}$ has purely discrete spectrum in $\left[G\left(H_{L}^{m}\right), G\left(H_{L}^{m}\right)+m\right)$. Moreover, Lemmas 3.6 and 3.9 yield that $H^{m}$ has purely discrete spectrum in $\left[G\left(H^{m}\right), G\left(H^{m}\right)+m\right)$. Thus the lemma follows.

3.3. Overlap and existence of ground states. In this subsection we show that there exist the ground states of $H$. Let us denote a ground state of $H^{m}$ by $\Omega_{m}$.

Lemma 3.11. There exists a constant $\delta(e)$ independent of $m$ so that $\left\|N^{1 / 2} \Omega_{m}\right\| \leq$ $\delta(e)\left\|\Omega_{m}\right\|$. 
Proof. For simplicity in this proof, we denote $\Omega_{m}$ by $\Omega, I \otimes a^{\sharp r}(f)$ by $a^{\sharp r}(f)$ and $p_{\mu}^{j} \otimes I$ by $p_{\mu}^{j}$. Note that since $\Omega$ is an eigenvector of $H^{m}$, we have $\Omega \in \mathcal{D}_{m}$. In particular, $\Omega \in D\left(a^{\sharp r}(f)\right), f \in \mathbf{M}_{2}$, and $\Omega \in D\left(p_{\mu}^{j}\right), j=1, \ldots, N, \mu=1, \ldots, d$. For $\Psi \in C_{0}^{\infty}\left(\mathbb{R}^{d}\right) \widehat{\otimes}\left[\mathcal{F}^{\infty} \cap D\left(H_{\mathrm{f}}^{3 / 2}\right)\right]$, one can show that, for $f \in \mathbf{M}_{4}$,

$$
\begin{aligned}
& \left(a^{r}(f) \Psi,\left(H^{m}-G\left(H^{m}\right)\right) a^{r}(f) \Psi\right)=-\left(a^{r}(f) \Psi, a^{r}((\omega+m) f) \Psi\right) \\
& \quad-\frac{e}{\sqrt{2}} \sum_{j=1}^{N} \sum_{\mu=1}^{d}\left(a^{r}(f) \Psi,\left(\bar{f}, \frac{\hat{\rho} e_{\mu}^{r} e^{-i k x^{j}}}{\sqrt{\omega}}\right) p_{\mu}^{j} \Psi\right) \\
& \quad-\frac{e^{2}}{\sqrt{2}} \sum_{j=1}^{N} \sum_{\mu=1}^{d}\left(a^{r}(f) \Psi,\left(\bar{f}, \frac{\hat{\rho} e_{\mu}^{r} e^{-i k x^{j}}}{\sqrt{\omega}}\right) A_{\mu}\left(x^{j}\right) \Psi\right) \\
& +\left(a^{r}(f) \Psi, a^{r}(f)\left(H^{m}-G\left(H^{m}\right)\right) \Psi\right) \geq 0 .
\end{aligned}
$$

It is possible to take the sequence $\Psi_{n} \in C_{0}^{\infty}\left(\mathbb{R}^{d}\right) \widehat{\otimes}\left[\mathcal{F}^{\infty} \cap D\left(H_{\mathrm{f}}^{3 / 2}\right)\right]$ so that $s-\lim _{n \rightarrow \infty} \Psi_{n}=\Omega$ and $s-\lim _{n \rightarrow \infty} H^{m} \Psi_{n}=H^{m} \Omega$, since $C_{0}^{\infty}\left(\mathbb{R}^{d}\right) \widehat{\otimes}\left[\mathcal{F}^{\infty} \cap D\left(H_{\mathrm{f}}^{3 / 2}\right)\right]$ is a core for $H^{m}$ by Proposition 3.3 (2) and Proposition 3.1 (5). Note that $a^{\sharp r}(g) \Psi_{n} \rightarrow a^{\sharp r}(g) \Omega, g \in \mathbf{M}_{2}$, and $p_{\mu}^{j} \Psi_{n} \rightarrow p_{\mu}^{j} \Omega$ as $n \rightarrow \infty$ strongly. Thus we have, for $f \in \mathbf{M}_{4}$,

$$
\begin{array}{r}
-\left(a^{r}(f) \Omega, a^{r}((\omega+m) f) \Omega\right)-\frac{e}{\sqrt{2}} \sum_{j=1}^{N} \sum_{\mu=1}^{d}\left(a^{r}(f) \Omega,\left(\bar{f}, \frac{\hat{\rho} e_{\mu}^{r} e^{-i k x^{j}}}{\sqrt{\omega}}\right) p_{\mu}^{j} \Omega\right) \\
-\frac{e^{2}}{\sqrt{2}} \sum_{j=1}^{N} \sum_{\mu=1}^{d}\left(a^{r}(f) \Omega,\left(\bar{f}, \frac{\hat{\rho} e_{\mu}^{r} e^{-i k x^{j}}}{\sqrt{\omega}}\right) A_{\mu}\left(x^{j}\right) \Omega\right) \geq 0 .
\end{array}
$$

Let $\left\{f_{l}\right\}_{l=1}^{\infty}$ be a complete orthogonal system of $L^{2}\left(\mathbb{R}^{d}\right)$ so that $f_{l} / \sqrt{\omega+m} \in \mathbf{M}_{4}$. Putting $f_{l} / \sqrt{\omega+m}$ 's in (3.20), and summing with respect to $l$ and $r$, one can see that (refer to see [11, Lemma 4.2])

$$
\begin{aligned}
\left\|N^{1 / 2} \Omega\right\|^{2} & +\sum_{j=1}^{N} \sum_{\mu=1}^{d} \sum_{r=1}^{d-1} \frac{e}{\sqrt{2}}\left(a^{r}\left(\frac{\overline{\hat{\rho}} e_{\mu}^{r} e^{i k x^{j}}}{\sqrt{\omega}(\omega+m)}\right) \Omega, p_{\mu}^{j} \Omega\right) \\
& +\sum_{j=1}^{N} \sum_{\mu=1}^{d} \sum_{r=1}^{d-1} \frac{e^{2}}{\sqrt{2}}\left(a^{r}\left(\frac{\overline{\hat{\rho}} e_{\mu}^{r} e^{i k x^{j}}}{\sqrt{\omega}(\omega+m)}\right) \Omega, A_{\mu}\left(x^{j}\right) \Omega\right) \leq 0 .
\end{aligned}
$$

Thus we have by (2.8)

$$
\begin{aligned}
\left\|N^{1 / 2} \Omega\right\|^{2} \leq & \sum_{j=1}^{N} \sum_{\mu=1}^{d} \frac{(d-1) e}{\sqrt{2}}\|\hat{\rho}\|_{-3}\left\|N^{1 / 2} \Omega\right\|\left\|p_{\mu}^{j} \Omega\right\| \\
& +\frac{d(d-1) N e^{2}}{2}\|\hat{\rho}\|_{-3}\|\hat{\rho}\|_{-1}\left\|N^{1 / 2} \Omega\right\|\left(\left\|N^{1 / 2} \Omega\right\|+\left\|(N+I)^{1 / 2} \Omega\right\|\right) .
\end{aligned}
$$

Then

$$
\begin{aligned}
& \left(1-d(d-1) N e^{2}\|\hat{\rho}\|_{-3}\|\hat{\rho}\|_{-1}\right)\left\|N^{1 / 2} \Omega\right\| \\
& \leq \sum_{j=1}^{N} \sum_{\mu=1}^{d} \frac{(d-1) e}{\sqrt{2}}\|\hat{\rho}\|_{-3}\left\|p_{\mu}^{j} \Omega\right\|+\frac{d(d-1) N e^{2}}{2}\|\hat{\rho}\|_{-3}\|\hat{\rho}\|_{-1}\|\Omega\|
\end{aligned}
$$


There exists a constant, $\mathcal{A}$, which is independent of $m$, so that

$$
\begin{aligned}
\left\|p_{\mu}^{j} \Omega\right\|^{2} & \leq\|\Omega\|\left\|\left(p_{\mu}^{j}\right)^{2}\left(H_{0}-i\right)^{-1}\right\|\left\|\left(H_{0}-i\right)\left(H^{m}-i\right)^{-1}\right\|\left\|\left(H^{m}-i\right) \Omega\right\| \\
& \leq \mathcal{A}\left(\left|G\left(H^{m}\right)\right|+1\right)\|\Omega\|^{2} .
\end{aligned}
$$

By (3.9) and (2.24) ${ }^{3}$

$$
\mathcal{A}:=\left(\frac{2\left(e M_{\mathrm{I}}+e^{2} M_{\mathrm{II}}+1\right)|\mathbf{g}|+2}{1-e M_{\mathrm{I}}-e^{2} M_{\mathrm{II}}}\right) \times\left(\frac{2 \alpha(b(\epsilon)+\epsilon \beta+2|\mathbf{g}|+1)}{1-\alpha \epsilon}+\beta\right),
$$

where $\alpha, \beta, \epsilon$ and $b(\epsilon)$ are in (2.3) . By (2.19) and diamagnetic inequalities [23] 4 we see that

$$
\mathbf{g}\|\Psi\|^{2} \leq\left(\Psi, H^{m} \Psi\right) \leq \widetilde{\mathcal{B}}\left(\Psi, H_{0}^{m} \Psi\right)+\widetilde{\mathcal{C}}\|\Psi\|^{2},
$$

where $\widetilde{\mathcal{B}}:=1+e M_{\mathrm{I}}^{\prime}+e^{2} M_{\mathrm{II}}^{\prime}$ and $\widetilde{\mathcal{C}}:=e \alpha_{\mathrm{I}}+e^{2} \alpha_{\mathrm{II}}$. Then we have

$$
\left|G\left(H^{m}\right)\right| \leq \max \left\{|\mathbf{g}|,\left|\mathbf{g}+\frac{e \sqrt{2} A\left(\mathbf{g}+a\left(\epsilon^{\prime}\right)\right)}{1-\epsilon^{\prime}}+\frac{\widetilde{B}\|\hat{\rho}\|_{-1}}{4} e^{2}\right|\right\},
$$

where $a\left(\epsilon^{\prime}\right)$ and $\epsilon^{\prime}$ are in (2.5). We put the right-hand side of (3.21) by $\mathcal{B}$. Hence we have

$$
\begin{aligned}
\frac{\left\|N^{1 / 2} \Omega\right\|}{\|\Omega\|} & \leq \frac{e d(d-1) N\|\hat{\rho}\|_{-3}}{1-d(d-1) N e^{2}\|\hat{\rho}\|_{-3}\|\hat{\rho}\|_{-1}}\left\{\frac{1}{\sqrt{2}} \sqrt{\mathcal{A}\left(\left|G\left(H^{m}\right)\right|+1\right)}+\frac{e\|\hat{\rho}\|_{-1}}{2}\right\} \\
& \leq \frac{e d(d-1) N\|\hat{\rho}\|_{-3}}{1-d(d-1) N e^{2}\|\hat{\rho}\|_{-3}\|\hat{\rho}\|_{-1}}\left\{\frac{1}{\sqrt{2}} \sqrt{\mathcal{A}(\mathcal{B}+1)}+\frac{e\|\hat{\rho}\|_{-1}}{2}\right\} . \\
& \equiv \delta(e) .
\end{aligned}
$$

Since the right-hand side of (3.22) is independent of $m$, the proof is complete.

Without loss of generality, we can assume that $\left\|\Omega_{m}\right\|=1$. Then one can find a subsequence $\left\{m_{j}\right\}_{j=1}^{\infty}, m_{1}>m_{2}>\cdots \rightarrow 0$, so that $\Omega_{g}=w-\lim _{j \rightarrow \infty} \Omega\left(m_{j}\right)$ exists. We give a general lemma.

Lemma 3.12 (11, Lemma 4.9]). Let $T_{n}, n=1,2, \ldots$, and $T$ be self-adjoint operators on Hilbert space $K$ having common core $D$ such that, for all $\phi \in D, T_{n} \phi \rightarrow T \phi$ as $n \rightarrow \infty$. Let $\phi_{n}$ be a normalized ground state of $T_{n}$ with ground state energy $E_{n}$ so that $E=\lim _{n \rightarrow \infty} E_{n}$ and $w-\lim _{n \rightarrow \infty} \phi_{n}=\phi \neq 0$. Then $\phi$ is a ground state of $T$ with ground state energy $E$.

Lemma 3.13. We see that (1) $\lim _{m \rightarrow 0} G\left(H^{m}\right)=G(H)$ and $(2) \Omega_{g} \neq 0$.

Proof. The idea of the proof is similar to that of [11]. Domain $C_{0}^{\infty}\left(\mathbb{R}^{d N}\right) \widehat{\otimes}\left[\mathcal{F}^{\infty} \cap\right.$ $\left.D\left(H_{\mathrm{f}}\right)\right]$ is a common core for family $H^{m}, m \geq 0$, by Proposition $3.3(2)$ and Proposition 3.1 (5). It is straightforward to see that, on $C_{0}^{\infty}\left(\mathbb{R}^{d N}\right) \widehat{\otimes}\left[\mathcal{F}^{\infty} \cap D\left(H_{\mathrm{f}}\right)\right], H^{m} \rightarrow H$ as $m \rightarrow 0$ strongly, which implies that $H^{m}$ converges to $H$ in the strong resolvent sense. Hence

$$
\limsup _{m \rightarrow 0} G\left(H^{m}\right) \leq G(H)
$$

\footnotetext{
${ }^{3}$ Generally, if $\|T \Psi\|_{\mathcal{K}} \leq a\|S \Psi\|_{\mathcal{K}}+b\|\Psi\|_{\mathcal{K}}, \Psi \in D(S) \subset D(T)$, then $\left\|(T-w)(S-z)^{-1} \Psi\right\|_{\mathcal{K}} \leq$ $K(a, b)\|\Psi\|_{\mathcal{K}}$, where $z, w \in \mathbb{C} \backslash \mathbb{R}, K(a, b):=a+b /|\Im z|+|w| /|\Im z|$.

${ }^{4}$ Unless using diamagnetic inequalities we have from (2.20) $\left(1-e M^{\prime}\right)\left(\Psi, H_{0}^{m} \Psi\right) \leq\left(\Psi, H^{m} \Psi\right)+$ $\left(e \alpha_{\mathrm{I}}+e^{2} \alpha_{\mathrm{II}}\right)\|\Psi\|^{2}$.
} 
But, from Proposition 3.1 (1) it follows that $G\left(H^{m}\right) \geq G(H)+m\left\|N^{1 / 2} \Omega_{m}\right\|^{2}$. Hence, from Lemma 3.11 it follows that

$$
\liminf _{m \rightarrow 0} G\left(H^{m}\right)=G(H) .
$$

Thus (3.23) and (3.24) imply (1). We shall now prove (2). In order to do so, we prove that, for sufficiently large $\Sigma$, there exists a positive constant, $\epsilon$, so that

$$
\left\|E_{H_{\mathrm{el}}}([\mathrm{g}, \Sigma)) \otimes E_{H_{\mathrm{f}}}(\{0\}) \Omega_{g}\right\| \geq \epsilon .
$$

For simplicity, we put $P:=E_{H_{\mathrm{el}}}([\mathbf{g}, \Sigma)) \otimes E_{H_{\mathrm{f}}}(\{0\}), Q:=E_{H_{\mathrm{el}}}([\Sigma, \infty)) \otimes E_{H_{\mathrm{f}}}(\{0\})$. Then

$$
\left(\Omega_{m}, P \Omega_{m}\right) \geq 1-\left\|N^{1 / 2} \Omega_{m}\right\|^{2}-\left(\Omega_{m}, Q \Omega_{m}\right) .
$$

One major point of the proof of (3.25) is to suppress $\left(\Omega_{m}, Q \Omega_{m}\right)$ by a sufficiently small constant. It is easily seen that

$$
\left(\Omega_{m}, Q\left(H_{\mathrm{el}} \otimes I-G\left(H^{m}\right)\right) \Omega_{m}\right)=-\left(\Omega_{m}, Q\left(e H_{\mathrm{I}}+e^{2} H_{\mathrm{II}}\right) \Omega_{m}\right) .
$$

We have from (2.13) and (3.9)

$$
\left\|H_{\mathrm{I}} \Omega_{m}\right\| \leq M_{\mathrm{I}}\left(c_{2}\left\|H^{m} \Omega_{m}\right\|+1+c_{2}\right), \quad\left\|H_{\mathrm{II}} \Omega_{m}\right\| \leq M_{\mathrm{II}}\left(c_{2}\left\|H^{m} \Omega_{m}\right\|+1+c_{2}\right) .
$$

Put $M:=\max \left\{M_{\mathrm{I}}\left(1+c_{2}\right), M_{\mathrm{II}}\left(1+c_{2}\right)\right\}$. Thus it follows from (3.27) that

$$
\begin{aligned}
(\Sigma- & \left.G\left(H^{m}\right)\right)\left(\Omega_{m}, Q \Omega_{m}\right) \leq\left(Q \Omega_{m},\left(e H_{\mathrm{I}}+e^{2} H_{\mathrm{II}}\right) \Omega_{m}\right) \\
& \leq e\left\|Q \Omega_{m}\right\|\left\|H_{\mathrm{I}} \Omega_{m}\right\|+e^{2}\left\|Q \Omega_{m}\right\|\left\|H_{\mathrm{II}} \Omega_{m}\right\| \\
& \leq\left\{\left(e M+e^{2} M\right)\left\|H^{m} \Omega_{m}\right\|+\left(e M+e^{2} M\right)\right\}\left\|Q \Omega_{m}\right\| .
\end{aligned}
$$

Thus we have

$$
\left(\Omega_{m}, Q \Omega_{m}\right) \leq\left\{\frac{\left(1+\left|G\left(H^{m}\right)\right|\right)\left(e M+e^{2} M\right)}{\Sigma-G\left(H^{m}\right)}\right\}^{2},
$$

which implies, together with Lemma 3.11 and $(\underline{3.26})$, that

$$
\left(\Omega_{m}, P \Omega_{m}\right) \geq 1-\delta(e)^{2}-\left\{\frac{\left(1+\left|G\left(H^{m}\right)\right|\right)\left(e M+e^{2} M\right)}{\Sigma-G\left(H^{m}\right)}\right\}^{2} .
$$

Since $P$ is finite rank, taking the subsequence $\left\{m_{j}\right\}_{j=1}^{\infty}$, we see that the left-hand side of (3.28) converges to $\left(\Omega_{g}, P \Omega_{g}\right)$ as $j \rightarrow \infty$. Thus from Lemma 3.13 it follows that

$$
\left(\Omega_{g}, P \Omega_{g}\right) \geq 1-\delta(e)^{2}-\left\{\frac{(1+|G(H)|)\left(e M+e^{2} M\right)}{\Sigma-G(H)}\right\}^{2} .
$$

Since by Hypothesis $\mathbf{1}, 1-\delta(e)^{2}>0$, taking $\Sigma$ sufficiently large, we get (2).

Theorem 3.14. The vector $\Omega_{g}$ is a ground state of $H$. Moreover, assume that $H_{\mathrm{el}}$ has a normalized unique ground state $\Psi$ and put $\Omega=\Psi \otimes \Omega$. Then

$$
\lim _{e \rightarrow 0}\left\|\Omega-\Omega_{g}\right\|=0 .
$$

Proof. From Lemmas 3.12 and 3.13 the first statement follows. Take $\Sigma>\mathrm{g}$ so that the dimension of the range of $P=E_{H_{\mathrm{el}}}([\mathbf{g}, \Sigma))$ is one. Taking $e$ sufficiently small, 
since $\lim _{e \rightarrow 0} G(H)=\mathbf{g}$, we see that (3.29) follows for such $\Sigma$. Then since $H_{0}$ has a unique ground state $\Omega$, we have

$$
\left(\Omega_{g}, \Omega\right)^{2}=\left(\Omega_{g}, P \Omega_{g}\right) \geq 1-\delta(e)^{2}-\left\{\frac{(1+|G(H)|)\left(e M+e^{2} M\right)}{\Sigma-G(H)}\right\}^{2} .
$$

The right-hand side of (3.31) goes to one as $e \rightarrow 0$. Since $\left\|\Omega-\Omega_{g}\right\|^{2}=2-2 \Re\left(\Omega_{g}, \Omega\right)$, (3.30) follows.

\section{SCATtERING THEORY}

Asymptotic annihilation operators $a_{ \pm}^{r}(h)$, and asymptotic creation operators $a_{ \pm}^{\dagger r}(h)$, are defined by the strong limits as time goes to $\pm \infty$ of the operators of the form

$$
a_{t}^{\sharp r}(h):=e^{i t H} e^{-i t H_{0}}\left(I \otimes a^{\sharp r}(h)\right) e^{i t H_{0}} e^{-i t H},
$$

on a dense domain in $\mathcal{H}$ for some $h \in L^{2}\left(\mathbb{R}^{d}\right)$. The first problem to be solved is to show the strong convergence of $a_{t}^{\sharp r}(h)$ as $t \rightarrow \pm \infty$ on a dense domain, and the second is to establish several algebraic relations between $a_{ \pm}^{r}(h)$ and $H$. Finally, using $a_{ \pm}^{r}(h)$ and the ground states of $H$, we specify the absolutely continuous spectrum of $H$.

4.1. Asymptotic annihilation and creation operators. In this subsection, we just handle the case $a_{t}^{\dagger r}(h)$ as $t \rightarrow \infty$, the other cases, $a_{t}^{r}(h), t \rightarrow \pm \infty / a_{t}^{\dagger r}(h), t \rightarrow$ $-\infty$, are done quite similarly.

Lemma 4.1. Suppose that Hypothesis 1, 2 and 3 hold. Let $f \in \mathbf{M}_{4}$ and

$$
K_{\mu}^{r}\left(t, x^{j}, f\right):=\left(\frac{\hat{\rho} e_{\mu}^{r} e^{-i k x^{j}}}{\sqrt{2 \omega}}, e^{-i t \omega} f\right) .
$$

Then, for $\Psi \in D(H)$ and $T<t$,

$$
\begin{aligned}
& a_{t}^{\dagger r}(f) \Psi=a_{T}^{\dagger r}(f) \Psi \\
& \quad-i \sum_{j=1}^{N} \sum_{\mu=1}^{d} \int_{T}^{t} e^{i s H}\left(e K_{\mu}^{r}\left(s, x^{j}, f\right) \otimes I\right)\left\{p_{\mu}^{j} \otimes I-e A_{\mu}\left(x^{j}\right)\right\} e^{-i s H} \Psi d s,
\end{aligned}
$$

where integral (4.1) is the Bochner integral.

Proof. We divide the proof into three steps. In this proof, for notational simplicity, we abbreviate $X \otimes I$ and $I \otimes X$ by $X$, and summation over repeated $\mu$ and $j$ is understood.

STEP I. Let $\Psi$ and $\Phi$ be in $D\left(H_{\mathrm{f}}\right)$, and $f \in \mathbf{M}_{4}$. Then

$$
\left(H_{\mathrm{f}} \Psi, a^{\dagger r}(f) \Phi\right)-\left(a^{r}(\bar{f}) \Psi, H_{\mathrm{f}} \Phi\right)=\left(\Psi, a^{\dagger r}(\omega f) \Phi\right) .
$$

Proof. From (2.6) one can find sequences $\left\{\Psi_{n}\right\}_{n=1}^{\infty}$ and $\left\{\Phi_{n}\right\}_{n=1}^{\infty}$ so that $\Psi_{n}, \Phi_{n} \in$ $C^{\infty}\left(H_{\mathrm{f}}\right)$, and $a^{\sharp r}(g) \Psi_{n} \rightarrow a^{\sharp r}(g) \Psi, H_{\mathrm{f}} \Psi_{n} \rightarrow H_{\mathrm{f}} \Psi, a^{\sharp r}(g) \Phi_{n} \rightarrow a^{\sharp r}(g) \Phi, H_{\mathrm{f}} \Phi_{n} \rightarrow$ $H_{\mathrm{f}} \Phi$, for $g \in \mathbf{M}_{2}$, as $n \rightarrow \infty$. Since, for each $n$, $\left[H_{\mathrm{f}}, a^{\dagger r}(f)\right] \Psi_{n}$ is well defined and $\left(H_{\mathrm{f}} \Psi_{n}, a^{\dagger r}(f) \Phi_{n}\right)-\left(a^{r}(\bar{f}) \Psi_{n}, H_{\mathrm{f}} \Phi_{n}\right)=\left(\Psi_{n}, a^{\dagger r}(\omega f) \Phi_{n}\right)$, taking $n \rightarrow \infty$, we see that (4.2) follows. 
STEP II. Let $\Psi, \Phi \in D(H)$ and $f \in \mathbf{M}_{4}$. Then

$$
\frac{d}{d t}\left(\Psi, a_{t}^{\dagger r}(f) \Phi\right)=-i\left(e^{-i t H} \Psi, e K_{\mu}^{r}\left(t, x^{j}, f\right)\left(p_{\mu}^{j}-e A_{\mu}\left(x^{j}\right)\right) e^{-i t H} \Phi\right) .
$$

Proof. Since $\hat{\rho} / \sqrt{\omega} \in \mathbf{M}_{4},\left[A_{\mu}\left(x^{j}\right), a^{\dagger r}\left(e^{-i t \omega} f\right)\right]$ and $\left[A_{\mu}\left(x^{j}\right), a^{r}\left(e^{i t \omega} \bar{f}\right)\right]$ are well defined on $D(H)$. It is straightforward to obtain, on $D(H)$,

$$
\left[A_{\mu}\left(x^{j}\right), a^{\dagger r}\left(e^{-i t \omega} f\right)\right]=K_{\mu}^{r}\left(t, x^{j}, f\right), \quad\left[A_{\mu}\left(x^{j}\right), a^{r}\left(e^{i t \omega} \bar{f}\right)\right]=-\overline{K_{\mu}^{r}\left(t, x^{j}, f\right)} .
$$

We see that

$$
\begin{aligned}
\frac{1}{\epsilon}(\Psi, & \left.\left(a_{t+\epsilon}^{\dagger r}(f)-a_{t}^{\dagger r}(f)\right) \Phi\right) \\
= & \frac{1}{\epsilon}\left\{\left(\Psi, e^{i(t+\epsilon) H} a^{\dagger r}\left(e^{-i(t+\epsilon) \omega} f\right) e^{-i(t+\epsilon) H} \Phi\right)\right. \\
& \left.-\left(\Psi, e^{i t H} a^{\dagger r}\left(e^{-i t \omega} f\right) e^{-i t H} \Phi\right)\right\} \\
= & \left(a^{r}\left(e^{i(t+\epsilon) \omega} \bar{f}\right) e^{-i(t+\epsilon) H} \Psi, \frac{e^{-i(t+\epsilon) H}-e^{-i t H}}{\epsilon} \Phi\right) \\
& +\left(e^{-i(t+\epsilon) H} \Psi, \frac{a^{\dagger r}\left(e^{-i(t+\epsilon) \omega} f\right)-a^{\dagger r}\left(e^{-i t \omega} f\right)}{\epsilon} e^{-i t H} \Phi\right) \\
& +\left(\frac{e^{-i(t+\epsilon) H}-e^{-i t H}}{\epsilon} \Psi, a^{\dagger r}\left(e^{-i t \omega} f\right) e^{-i t H} \Phi\right)
\end{aligned}
$$

Since $e^{-i t H} \Psi \in D(H)$, taking $\epsilon \rightarrow 0$ in (4.4) and using (4.2), we have

$$
\begin{aligned}
\frac{d}{d t}(\Psi & \left., a_{t}^{\dagger r}(f) \Phi\right)=\left(a^{r}\left(e^{i t \omega} \bar{f}\right) e^{-i t H} \Psi,-i\left(e H_{\mathrm{I}}+e^{2} H_{\mathrm{II}}\right) e^{-i t H} \Phi\right) \\
& +\left(a^{r}\left(e^{i t \omega} \bar{f}\right) e^{-i t H} \Psi,-i H_{\mathrm{f}} e^{-i t H} \Phi\right) \\
& +\left(-i\left(e H_{\mathrm{I}}+e^{2} H_{\mathrm{II}}\right) e^{-i t H} \Psi, a^{\dagger r}\left(e^{-i t \omega} f\right) e^{-i t H} \Phi\right) \\
& +\left(-i H_{\mathrm{f}} e^{-i t H} \Psi, a^{\dagger r}\left(e^{-i t \omega} f\right) e^{-i t H} \Phi\right) \\
& +\left(e^{-i t H} \Psi, a^{\dagger r}\left(-i \omega e^{-i t \omega} f\right) e^{-i t H} \Phi\right) \\
& =i\left(H_{\mathrm{I}} e^{-i t H} \Psi, a^{\dagger r}\left(e^{-i t \omega} f\right) e^{-i t H} \Phi\right) \\
& -i\left(a^{r}\left(e^{i t \omega} \bar{f}\right) e^{-i t H} \Psi, H_{\mathrm{I}} e^{-i t H} \Phi\right) \\
& +i\left(H_{\mathrm{II}} e^{-i t H} \Psi, a^{\dagger r}\left(e^{-i t \omega} f\right) e^{-i t H} \Phi\right) \\
& -i\left(a^{r}\left(e^{i t \omega} \bar{f}\right) e^{-i t H} \Psi, H_{\mathrm{II}} e^{-i t H} \Phi\right) .
\end{aligned}
$$

Note that, on $D(H),\left[p_{\mu}^{j}, K_{\mu}^{j}\left(t, x^{j}, f\right)\right]=0$. Then we see that

$$
\begin{aligned}
& \left(H_{\mathrm{I}} e^{-i t H} \Psi, a^{\dagger r}\left(e^{-i t \omega} f\right) e^{-i t H} \Phi\right) \\
& =-\left(p_{\mu}^{j} e^{-i t H} \Psi, A_{\mu}\left(x^{j}\right) a^{\dagger r}\left(e^{-i t \omega} f\right) e^{-i t H} \Phi\right) \\
& =-\left(p_{\mu}^{j} e^{-i t H} \Psi, a^{\dagger r}\left(e^{-i t \omega} f\right) A_{\mu}\left(x^{j}\right) e^{-i t H} \Phi\right)-\left(p_{\mu}^{j} e^{-i t H} \Psi, K_{\mu}^{r}\left(t, x^{j}, f\right) e^{-i t H} \Phi\right) \\
& =\left(a^{r}\left(e^{i t \omega} \bar{f}\right) e^{-i t H} \Psi, H_{\mathrm{I}} e^{-i t H} \Phi\right)-\left(e^{-i t H} \Psi, K_{\mu}^{r}\left(t, x^{j}, f\right) p_{\mu}^{j} e^{-i t H} \Phi\right) .
\end{aligned}
$$


Moreover,

$$
\begin{aligned}
( & \left.H_{\mathrm{II}} e^{-i t H} \Psi, a^{\dagger r}\left(e^{-i t \omega} f\right) e^{-i t H} \Phi\right) \\
= & \frac{1}{2}\left(A_{\mu}\left(x^{j}\right) e^{-i t H} \Psi, a^{\dagger r}\left(e^{-i t \omega} f\right) A_{\mu}\left(x^{j}\right) e^{-i t H} \Phi\right) \\
& +\frac{1}{2}\left(A_{\mu}\left(x^{j}\right) e^{-i t H} \Psi, K_{\mu}^{r}\left(t, x^{j}, f\right) e^{-i t H} \Phi\right) \\
= & \frac{1}{2}\left(a^{r}\left(e^{i t \omega} \bar{f}\right) A_{\mu}\left(x^{j}\right) e^{-i t H} \Psi, A_{\mu}\left(x^{j}\right) e^{-i t H} \Phi\right) \\
& +\frac{1}{2}\left(A_{\mu}\left(x^{j}\right) e^{-i t H} \Psi, K_{\mu}^{r}\left(t, x^{j}, f\right) e^{-i t H} \Phi\right) \\
= & \frac{1}{2}\left(\overline{K_{\mu}^{r}\left(t, x_{j}\right)} e^{-i t H} \Psi, A_{\mu}\left(x^{j}\right) e^{-i t H} \Phi\right) \\
& +\frac{1}{2}\left(A_{\mu}\left(x^{j}\right) e^{-i t H} \Psi, K_{\mu}^{r}\left(t, x^{j}, f\right) e^{-i t H} \Phi\right) \\
& +\left(a^{r}\left(e^{i t \omega} \bar{f}\right) e^{-i t H} \Psi, H_{\mathrm{II}} e^{-i t H} \Phi\right) \\
= & \left(e^{-i t H} \Psi, K_{\mu}^{r}\left(t, x^{j}, f\right) A_{\mu}\left(x^{j}\right) e^{-i t H} \Phi\right) \\
& +\left(a^{r}\left(e^{i t \omega} \bar{f}\right) e^{-i t H} \Psi, H_{\mathrm{II}} e^{-i t H} \Phi\right) .
\end{aligned}
$$

Hence (4.3) follows from 4.5).

STEP III. The proof of Lemma 4.1.

From (2.21), 2.23) and 2.15) there exist a positive constant $\gamma$ so that, for $\Psi \in D(H)$,

$$
\left\|A_{\mu}\left(x^{j}\right) \Psi\right\| \leq \frac{1}{\sqrt{2}}\left(2\|\hat{\rho}\|_{-2}\left\|H_{\mathrm{f}}^{1 / 2} \Psi\right\|+\|\hat{\rho}\|_{-1}\|\Psi\|\right) \leq \gamma(\|H \Psi\|+\|\Psi\|) .
$$

Hence $\left(p_{\mu}^{j}-e A_{\mu}\left(x^{j}\right)\right) e^{-i t H} \Psi, \Psi \in D(H)$ is strongly continuous in $t$, which implies that

$$
e^{i t H} e K_{\mu}^{r}\left(t, x^{j}, f\right)\left(p_{\mu}^{j}-e A_{\mu}\left(x^{j}\right)\right) e^{-i t H} \Psi, \quad \Psi \in D(H)
$$

is strongly continuous in $t$. Then one can define the Bochner integral of (4.8) in $t$. Thus we have, by (4.3), for $\Psi, \Phi \in D(H)$,

$$
\begin{aligned}
& \left(\Psi, a_{t}^{\dagger r}(f) \Phi\right)-\left(\Psi, a_{T}^{\dagger r}(f) \Phi\right) \\
& \quad=\left(\Psi,-i \int_{T}^{t} e^{i s H} e K_{\mu}^{r}\left(s, x^{j}, f\right)\left(p_{\mu}^{j}-e A_{\mu}\left(x^{j}\right)\right) e^{-i s H} \Phi d s\right) .
\end{aligned}
$$

Since $D(H)$ is dense, (4.1) follows.

Remark 4.2. We may formally write (4.1) without domain arguments as

$$
a_{t}^{\dagger r}(f) \Psi=a_{T}^{\dagger r}(f) \Psi+\int_{T}^{t} i e^{i s H}\left[e H_{\mathrm{I}}+e^{2} H_{\mathrm{II}}, a^{\dagger r}\left(e^{-i s \omega} f\right)\right] e^{-i s H} \Psi d s .
$$

In fact, assuming that $\Psi \in D\left(H_{0}^{2}\right), \hat{\rho} \in \bigcap_{k=-2}^{3} \mathcal{M}_{k}$ and $h \in \bigcap_{k=-1}^{2} \mathcal{M}_{k}, 4.9$ can be verified.

We define a set of functions, $\mathcal{E}_{0}^{\infty}$, as follows: 
Definition 4.3. A function $f: \mathbb{R}^{d} \rightarrow \mathbb{R}$ is in $\mathcal{E}_{0}^{\infty}$, if, for all $h \in C_{0}^{\infty}\left(\mathbb{R}^{d} \backslash\{0\}\right)$,

$$
\lim _{t \rightarrow \infty} t^{\frac{d-1}{2}} \sup _{x \in \mathbb{R}^{d}}\left|\int_{\mathbb{R}^{d}} f(k) h(k) e^{i k x-i t \omega(k)} d k\right|<\infty .
$$

For mathematical convenience, we introduce the following hypothesis.

Hypothesis 4: The ultraviolet cut-off satisfies that $\hat{\rho} \in \mathcal{E}_{0}^{\infty}$ and $\partial_{\mu} \hat{\rho} \in \mathcal{E}_{0}^{\infty}, \mu=$ $1, \ldots, d$.

It is well-known [39] that if $\hat{\rho} \in C^{\infty}\left(\mathbb{R}^{d}\right)$, then $\hat{\rho} \in \mathcal{E}_{0}^{\infty}$ and $\partial_{\mu} \hat{\rho} \in \mathcal{E}_{0}^{\infty}, \mu=1, \ldots, d$.

Theorem 4.4. Suppose that Hypothesis 1, 2, 3 and 4 hold, and $f \in C_{0}^{\infty}\left(\mathbb{R}^{d} \backslash \mathcal{P}\right)$. Then, for $\Psi \in D(H)$, there exists the strong limit of $a_{t}^{\sharp r}(f) \Psi$ as $t \rightarrow \infty$.

Proof. By virtue of the integral representation (4.1), it suffices to show that, for $j=1, \ldots, N, r=1, \ldots, d-1, \mu=1, \ldots, d$, and $\Psi \in D(H)$,

$$
\left\|\left(e K_{\mu}^{r}\left(t, x^{j}, f\right) \otimes I\right)\left(p_{\mu}^{j} \otimes I-e A_{\mu}\left(x^{j}\right)\right) e^{-i t H} \Psi\right\| \in L^{1}([T, \infty), d t)
$$

with some $T$. Fix $j, r, \mu$. First we note that, by (2.22), (2.26), (2.28), (2.21), (2.23) and (2.15), there exists a positive constant $\alpha$ so that

$$
\begin{aligned}
\left\|\left(x_{\mu}^{j} p_{\mu}^{j} \otimes I\right) \Psi\right\| \leq & \alpha(\|H \Psi\|+\|\Psi\|), \\
\left\|x_{\mu}^{j} A_{\mu}\left(x^{j}\right) \Psi\right\| \leq & \frac{1}{\sqrt{2}}\left(2\|\hat{\rho}\|_{-2}\left\|\left(x_{\mu}^{j} \otimes H_{\mathrm{f}}^{1 / 2}\right) \Psi\right\|\right. \\
& \left.+\|\hat{\rho}\|_{-1}\left\|\left(x_{\mu}^{j} \otimes I\right) \Psi\right\|\right) \leq \alpha(\|H \Psi\|+\|\Psi\|) .
\end{aligned}
$$

We consider $\left(K_{\mu}^{r}\left(t, x^{j}, f\right) p_{\mu}^{j} \otimes I\right) e^{-i t H} \Psi$ and $\left(K_{\mu}^{r}\left(t, x^{j}, f\right) \otimes I\right) A_{\mu}\left(x^{j}\right) e^{-i t H} \Psi$, separately. Let $\lambda_{\mu}^{r}(k):=\sqrt{\omega(k)} f(k) e_{\mu}^{r}(k) /\left(\sqrt{2} k_{\mu}\right)$. Using

$$
e^{i t \omega(k)}=\frac{\omega(k)}{k_{\mu}} \frac{1}{i t} \frac{\partial}{\partial k_{\mu}} e^{i t \omega(k)}, \quad k \in \mathbb{R}^{d} \backslash\{0\},
$$

and integrating by parts using that the kernels are smooth enough, one sees that

$$
K_{\mu}^{r}\left(t, x^{j}, f\right)=\frac{1}{t} K_{\mu}^{(1)}\left(t, x^{j}, f\right)+\frac{1}{t} K_{\mu}^{(2)}\left(t, x^{j}, f\right) x_{\mu}^{j},
$$

where

$$
\begin{gathered}
K_{\mu}^{(1)}\left(t, x^{j}, f\right):=i \int_{\mathbb{R}^{d}} e^{-i t \omega(k)+i k x^{j}} \frac{\partial}{\partial k_{\mu}}\left(\hat{\rho}(-k) \lambda_{\mu}^{r}(k)\right) d k, \\
K_{\mu}^{(2)}\left(t, x^{j}, f\right):=\int_{\mathbb{R}^{d}} e^{-i t \omega(k)+i k x^{j}} \hat{\rho}(-k) \lambda_{\mu}^{r}(k) d k .
\end{gathered}
$$

Since $\lambda_{\mu}^{r} \in C_{0}^{\infty}\left(\mathbb{R}^{d} \backslash \mathcal{P}\right)$ and $\partial \lambda_{\mu}^{r} / \partial k_{\mu} \in C_{0}^{\infty}\left(\mathbb{R}^{d} \backslash \mathcal{P}\right)$ by 4.10 , there exists a positive constant, $K$, so that $\sup _{x^{j} \in \mathbb{R}^{d}}\left|K_{\mu}^{(i)}\left(t, x^{j}, f\right)\right| \leq K / t^{\frac{d-1}{2}}, i=1,2, t>0$. We have

$$
\left(K_{\mu}^{r}\left(t, x^{j}, f\right) p_{\mu}^{j} \otimes I\right) \Psi=\frac{1}{t}\left(K_{\mu}^{(1)}\left(t, x^{j}, f\right)+x_{\mu}^{j} K_{\mu}^{(2)}\left(t, x^{j}, f\right)\right)\left(p_{\mu}^{j} \otimes I\right) \Psi .
$$

Inequality (4.11) implies that

$$
\begin{aligned}
& \frac{1}{t}\left\|\left(x_{\mu}^{j} K_{\mu}^{(2)}\left(t, x^{j}, f\right) p_{\mu}^{j} \otimes I\right) e^{-i t H} \Psi\right\| \\
& \quad \leq \frac{1}{t}\left(\sup _{x^{j} \in \mathbb{R}^{d}}\left|K_{\mu}^{(2)}\left(t, x^{j}, f\right)\right|\right)\left\|\left(x_{\mu}^{j} p_{\mu}^{j} \otimes I\right) e^{-i t H} \Psi\right\| \\
& \quad \leq \frac{\alpha K}{t^{\frac{d+1}{2}}}(\|H \Psi\|+\|\Psi\|) .
\end{aligned}
$$


Thus we conclude that, since $d \geq 2$, the right-hand side of (4.13) is in $L^{1}([T, \infty), d t)$. For $K_{\mu}^{(1)}$, one can also see, by (4.6), that

$$
\frac{1}{t}\left\|\left(K_{\mu}^{(1)}\left(t, x^{j}, f\right) p_{\mu}^{j} \otimes I\right) e^{-i t H} \Psi\right\| \leq \frac{\alpha K}{t^{\frac{d+1}{2}}}(\|H \Psi\|+\|\Psi\|) \in L^{1}([T, \infty), d t) .
$$

Hence we have $\left\|\left(K_{\mu}^{r}\left(t, x^{j}, f\right) p_{\mu}^{j} \otimes I\right) e^{-i t H} \Psi\right\| \in L^{1}([T, \infty), d t)$. Next we have $\left(K_{\mu}^{r}\left(t, x^{j}, f\right) \otimes I\right) A_{\mu}\left(x^{j}, \hat{\rho}\right) \Psi=\frac{1}{t} A_{\mu}\left(x^{j}\right)\left(K_{\mu}^{(1)}\left(t, x^{j}, f\right)+x_{\mu}^{j} K_{\mu}^{(2)}\left(t, x^{j}, f\right)\right) \otimes I \Psi$.

From (4.12) it follows that

$$
\begin{aligned}
& \frac{1}{t}\left\|A_{\mu}\left(x^{j}\right)\left(x_{\mu}^{j} K_{\mu}^{(2)}\left(t, x^{j}, f\right) \otimes I\right) e^{-i t H} \Psi\right\| \\
& \quad \leq \frac{1}{t^{\frac{d+1}{2}}}\left(\sup _{x^{j} \in \mathbb{R}^{d}}\left|K_{\mu}^{(2)}\left(t, x^{j}, f\right)\right|\right)\left\|\left(x_{\mu}^{j} \otimes I\right) A_{\mu}\left(x^{j}\right) e^{-i t H} \Psi\right\| \\
& \leq \frac{\alpha K}{t^{\frac{d+1}{2}}}(\|H \Psi\|+\|\Psi\|) .
\end{aligned}
$$

Also by (4.7) we have $t^{-1}\left\|A_{\mu}\left(x^{j}\right)\left(K_{\mu}^{(1)}\left(t, x^{j}, f\right) \otimes I\right) e^{-i t H} \Psi\right\| \in L^{1}([T, \infty), d t)$. Hence it follows that $\left\|\left(K_{\mu}^{r}\left(t, x^{j}, f\right) \otimes I\right) A_{\mu}\left(x^{j}\right) e^{-i t H} \Psi\right\| \in L^{1}([T, \infty), d t)$. Thus the theorem follows.

4.2. Algebraic relations. In this subsection, we study extensions and algebraic relations of $a_{ \pm}^{\sharp r}(h)$.

Lemma 4.5. Assume that Hypothesis 1, 2, 3 and 4 hold. Let $h \in C_{0}^{\infty}\left(\mathbb{R}^{d} \backslash \mathcal{P}\right)$ and $\Psi, \Phi \in D(H)$. Then $\left(\Psi, a_{ \pm}^{r}(h) \Phi\right)=\left(a_{ \pm}^{\dagger r}(\bar{h}) \Psi, \Phi\right)$.

Proof. Since $\lim _{t \rightarrow \pm \infty}\left(\Psi, a_{t}^{r}(h) \Phi\right)=\lim _{t \rightarrow \pm \infty}\left(a_{t}^{\dagger r}(\bar{h}) \Psi, \Phi\right)$, the desired results follow easily.

By Lemma 4.5, $a_{ \pm}^{\sharp r}(h), h \in C_{0}^{\infty}\left(\mathbb{R}^{d} \backslash \mathcal{P}\right)$, are closable. We write their closures as the same symbols.

Lemma 4.6. Assume that Hypothesis 1, 2, 3 and 4 hold. Let $\Psi \in D(H)$ and $h \in C_{0}^{\infty}\left(\mathbb{R}^{d} \backslash \mathcal{P}\right)$. Then there exists positive constant $\alpha$ so that

$$
\left\|a_{ \pm}^{\sharp r}(h) \Psi\right\| \leq 2 \alpha\|h\|_{-1}\left\||H|^{1 / 2} \Psi\right\|+\left(2 \alpha\|h\|_{-1}+\|h\|_{0}\right)\|\Psi\| .
$$

Proof. By (2.27), (2.24) and Remark 2.5(2), we see that there exists $\alpha$ so that

$$
\left\|I \otimes H_{\mathrm{f}}^{1 / 2} \Psi\right\| \leq \alpha\left(\left\||H|^{1 / 2} \Psi\right\|+\|\Psi\|\right), \quad \Psi \in D\left(|H|^{1 / 2}\right) .
$$

Since $\left\|a_{ \pm}^{\sharp r}(h) \Psi\right\|=\lim _{t \rightarrow \pm \infty}\left\|a_{t}^{\sharp r}(h) \Psi\right\| \leq \lim _{t \rightarrow \pm \infty} 2\|h\|_{-1}\left\|I \otimes H_{\mathrm{f}}^{1 / 2} e^{-i t H} \Psi\right\|+$ $\|h\|_{0}\|\Psi\|$, combining with (4.15), we have (4.14).

It is immediately seen that $a_{ \pm}^{\sharp r}(h), h \in C_{0}^{\infty}\left(\mathbb{R}^{d} \backslash \mathcal{P}\right)$, extends to $h \in \mathbf{M}_{2}$ by (4.14). Since the extended operators $a_{ \pm}^{\sharp r}(h), h \in \mathbf{M}_{2}$, also have densely defined adjoints, they are closable. We write their closures as the same symbols. Moreover, from Lemma 4.6] it follows that $D\left(|H|^{1 / 2}\right) \subset D\left(a_{ \pm}^{\sharp r}(h)\right), h \in \mathbf{M}_{2}$, by the closedness of $a_{ \pm}^{\sharp r}(h)$. 
Lemma 4.7. Assume that Hypothesis 1, 2, 3 and 4 hold. Let $f \in \mathbf{M}_{2}$ and $g \in \mathbf{M}_{4}$. Then there exist positive constants $\alpha$ and $\beta$ so that, for $\Psi \in D(H)$,

$$
\left\|a_{ \pm}^{\sharp s}(f) a_{ \pm}^{\sharp r}(g) \Psi\right\| \leq\left(\frac{\alpha A}{2}+\beta B\right)\|H \Psi\|+\left(\frac{(3 \alpha+2) A}{2}+(\beta+1) B\right)\|\Psi\|,
$$

where $A:=\mathbf{k}\left(\|f\|_{-1}+\|f\|_{0}\right)\left(\|g\|_{1}+\|g\|_{2}\right)$ and $B:=\left(\|f\|_{-1}+\|f\|_{0}\right)\left(\|g\|_{-1}+\|g\|_{0}\right)$ with $\mathbf{k}$ in 2.7.

Proof. First we suppose that $f, g \in C_{0}^{\infty}\left(\mathbb{R}^{d} \backslash \mathcal{P}\right)$. By (2.11) and (2.15) one can see that there exists positive constant $\beta$ so that $\left\|I \otimes H_{\mathrm{f}} \Psi\right\| \leq \beta(\|H \Psi\|+\|\Psi\|), \Psi \in$ $D(H)$. By (2.9) and (4.15), we see that, with $\alpha$ in (4.15),

$$
\begin{aligned}
\left|\left(\Phi, a_{t}^{\dagger s}(f) a_{t}^{\dagger r}(h) \Psi\right)\right| \leq & \left\{A\left\|I \otimes H_{\mathrm{f}}^{1 / 2} e^{-i t H} \Psi\right\|\right. \\
& \left.+B\left\|I \otimes H_{\mathrm{f}} e^{-i t H} \Psi\right\|+(A+B)\|\Psi\|\right\}\|\Phi\| \\
\leq\left\{\alpha A\left\||H|^{1 / 2} \Psi\right\|\right. & +\beta B\|H \Psi\|+(\alpha A+\beta B+A+B)\|\Psi\|\}\|\Phi\|,
\end{aligned}
$$

Moreover, we have $\lim _{t \rightarrow \pm \infty}\left(\Phi, a_{t}^{\dagger s}(f) a_{t}^{\dagger r}(g) \Psi\right)=\left(a_{ \pm}^{s}(\bar{f}) \Phi, a_{ \pm}^{\dagger r}(g) \Psi\right)$. Thus

$$
\begin{aligned}
& \left|\left(a_{ \pm}^{s}(\bar{f}) \Phi, a_{ \pm}^{\dagger r}(g) \Psi\right)\right| \\
& \quad \leq\left\{\left(\frac{\alpha A}{2}+\beta B\right)\|H \Psi\|+\left(\frac{(3 \alpha+2) A}{2}+(\beta+1) B\right)\|\Psi\|\right\}\|\Phi\| .
\end{aligned}
$$

For $f \in \mathbf{M}_{2}$ and $g \in \mathbf{M}_{4}$, one can take $g_{n} \in C_{0}^{\infty}\left(\mathbb{R}^{d} \backslash \mathcal{P}\right)$, so that $g_{n} \rightarrow g$ as $n \rightarrow \infty$ in $\mathcal{M}_{-1}, \mathcal{M}_{0}, \mathcal{M}_{1}$ and $\mathcal{M}_{2}$, and $f_{n} \in C_{0}^{\infty}\left(\mathbb{R}^{d} \backslash \mathcal{P}\right)$ so that $f_{n} \rightarrow f$ as $n \rightarrow \infty$ in $\mathcal{M}_{-1}$ and $\mathcal{M}_{0}$. Then

$$
\begin{aligned}
& \left|\left(a_{ \pm}^{s}\left(\overline{f_{n}}\right) \Phi, a_{ \pm}^{\dagger r}\left(g_{n}\right) \Psi\right)\right| \\
& \quad \leq\left\{\left(\frac{\alpha A_{n}}{2}+\beta B_{n}\right)\|H \Psi\|+\left(\frac{(3 \alpha+2) A_{n}}{2}+(\beta+1) B_{n}\right)\|\Psi\|\right\}\|\Phi\|,
\end{aligned}
$$

where $A_{n}$ and $B_{n}$ are defined by $A$ and $B$ with $f$ and $g$ replaced by $f_{n}$ and $g_{n}$, respectively. Both sides of (4.18) converge to both sides of (4.17) as $n \rightarrow \infty$, respectively, which implies that $a_{ \pm}^{\dagger r}(g) \Psi \in D\left(a_{ \pm}^{\dagger s}(f)\right)$ and that (4.16) follows.

Lemma 4.8. Assume that Hypothesis 1, 2, 3 and 4 hold. We see that, for $f, g \in$ $\mathbf{M}_{4}$, the following commutation relations hold on $D(H)$;

$$
\begin{aligned}
{\left[a_{ \pm}^{s}(f), a_{ \pm}^{\dagger r}(g)\right] } & =\delta_{s, r}(\bar{f}, g), \\
{\left[a_{ \pm}^{s}(f), a_{ \pm}^{r}(g)\right] } & =0 .
\end{aligned}
$$

Proof. Note that, by Lemma 4.7, for $f, g \in \mathbf{M}_{4}, a_{ \pm}^{\sharp s}(f) a_{ \pm}^{\sharp r}(g) \Psi, \Psi \in D(H)$, is well defined. Assume first that $f, g \in C_{0}^{\infty}\left(\mathbb{R}^{d} \backslash \mathcal{P}\right)$. We see that

$$
\begin{aligned}
& \left(\Psi,\left[a_{ \pm}^{s}(f), a_{ \pm}^{\dagger r}(g)\right] \Phi\right)=\left(\Psi, a_{ \pm}^{s}(f) a_{ \pm}^{\dagger r}(g) \Phi\right)-\left(\Psi, a_{ \pm}^{\dagger r}(g) a_{ \pm}^{s}(f) \Phi\right) \\
= & \lim _{t \rightarrow \pm \infty}\left\{\left(a_{t}^{\dagger s}(\bar{f}) \Psi, a_{t}^{\dagger r}(g) \Phi\right)-\left(a_{t}^{r}(\bar{g}) \Psi, a_{t}^{s}(f) \Phi\right)\right\}=\delta_{s, r}(\bar{f}, g)(\Psi, \Phi) .
\end{aligned}
$$

Since $D(H)$ is dense, (4.19) follows for such $f$ and $g$. For approximate arguments similar to those in Lemma 4.7, one can get (4.19) for $f, g \in \mathbf{M}_{4}$. (4.20) can be treated quite similarly. 
Lemma 4.9. Assume that Hypothesis 1,2,3 and 4 hold. Let $h \in \mathbf{M}_{2}$ and $\Psi \in$ $D\left(|H|^{1 / 2}\right)$. Then

$$
\begin{aligned}
& e^{i t H} a_{ \pm}^{\dagger r}(h) \Psi=a_{ \pm}^{\dagger r}\left(e^{i t \omega} h\right) e^{i t H} \Psi, \\
& e^{i t H} a_{ \pm}^{r}(h) \Psi=a_{ \pm}^{r}\left(e^{-i t \omega} h\right) e^{i t H} \Psi .
\end{aligned}
$$

Proof. First, assume $h \in C_{0}^{\infty}\left(\mathbb{R}^{d} \backslash \mathcal{P}\right)$. By the definition of $a_{ \pm}^{\sharp r}(h)$, we can easily see (4.21) and (4.22). Next one can easily extend (4.21) and (4.22) to $h \in \mathbf{M}_{2}$ by (4.14).

Lemma 4.10. Assume that Hypothesis 1, 2, 3 and 4 hold. Let $h \in \mathbf{M}_{4}$. Then $a_{ \pm}^{\sharp r}(h)$ maps $D\left(|H|^{3 / 2}\right)$ into $D(H)$, and the following commutation relation holds on $D\left(|H|^{3 / 2}\right)$;

$$
\begin{aligned}
& {\left[H, a_{ \pm}^{\dagger r}(h)\right]=a_{ \pm}^{\dagger r}(\omega h),} \\
& {\left[H, a_{ \pm}^{r}(h)\right]=-a_{ \pm}^{r}(\omega h) .}
\end{aligned}
$$

Proof. Note that $a_{ \pm}^{\dagger r}(h) H \Psi, \Psi \in D(H)$, is well-defined. By (4.14) we have

$$
\begin{gathered}
\left\|a_{ \pm}^{\dagger r}\left(e^{i t \omega} h\right)\left(\frac{e^{i t H}-I}{t}-i H\right) \Psi\right\| \leq 2 \alpha\|h\|_{-1}\left\|\left(\frac{e^{i t H}-I}{t}-i H\right)|H|^{1 / 2} \Psi\right\| \\
+\left(2 \alpha\|h\|_{-1}+\|h\|_{0}\right)\left\|\left(\frac{e^{i t H}-I}{t}-i H\right) \Psi\right\| .
\end{gathered}
$$

Moreover

$$
\begin{aligned}
& \left\|a_{ \pm}^{\dagger r}\left(\frac{e^{i t \omega}-1}{t} h-i \omega h\right) \Psi\right\| \leq 2 \alpha\left\|\frac{e^{i t \omega}-1}{t} h-i \omega h\right\|_{-1}\left\||H|^{1 / 2} \Psi\right\| \\
& +\left(2 \alpha\left\|\frac{e^{i t \omega}-1}{t} h-i \omega h\right\|_{-1}+\left\|\frac{e^{i t \omega}-1}{t} h-i \omega h\right\|_{0}\right)\|\Psi\| .
\end{aligned}
$$

Hence we see that the right-hand side of (4.21) is strongly differentiable in $t$ with $s-\frac{d}{d t} a_{ \pm}^{\dagger r}\left(e^{i t \omega} h\right) e^{i t H} \Psi=a_{ \pm}^{\dagger r}\left(i \omega e^{i t \omega} h\right) e^{i t H} \Psi+i a_{ \pm}^{\dagger r}\left(e^{i t \omega} h\right) H e^{i t H} \Psi$, which implies that $a_{ \pm}^{\dagger r}(h) \Psi \in D(H)$ and the left-hand side of (4.21) is strongly differentiable in $t$ with $i e^{i t H} H a_{ \pm}^{\dagger r}(h) \Psi=a_{ \pm}^{\dagger r}\left(i \omega e^{i t \omega} h\right) e^{i t H} \Psi+i a_{ \pm}^{\dagger r}\left(e^{i t \omega} h\right) H e^{i t H} \Psi$. Thus (4.23) follows. (4.24) is proven similarly to (4.23).

Lemma 4.11. Assume that Hypothesis 1, 2, 3 and 4 hold. Let $h \in \mathcal{M}_{-1} \cap \mathcal{M}_{0} \cap$ $\mathcal{M}_{1} \cap \ldots \cap \mathcal{M}_{2 n}, n \geq 1$. Then $a_{ \pm}^{\sharp r}(h)$ maps $D\left(|H|^{n+1 / 2}\right)$ into $D\left(H^{n}\right)$.

Proof. We prove the lemma for $a_{ \pm}^{\dagger r}(h)$ by an induction on $n$. The proof for the case of $a_{ \pm}^{r}(h)$ is similar. In the case of $n=1$, Lemma 4.10 leads to the lemma. Suppose that, in the cases of $n=1,2, . ., k \geq 1$, the lemma follows. Let $\Psi \in D\left(|H|^{k+1+1 / 2}\right)$. Hence, since $\Psi \in D\left(|H|^{3 / 2}\right)$, from Lemma 4.10 it follows that

$$
H a_{ \pm}^{\dagger r}(h) \Psi=a_{ \pm}^{\dagger r}(\omega h) \Psi+a_{ \pm}^{\dagger r}(h) H \Psi .
$$

Because of the fact that $H \Psi \in D\left(|H|^{k+1 / 2}\right)$ and $\Psi \in D\left(|H|^{k+1+1 / 2}\right) \subset D\left(|H|^{k+1 / 2}\right)$, by the assumption of the induction, the right-hand side of (4.25) is in $D\left(H^{k}\right)$. Hence $a_{ \pm}^{\dagger r}(h) \Psi \in D\left(H^{k+1}\right)$. Thus the lemma follows.

Lemma 4.12. Assume that Hypothesis 1, 2, 3 and 4 hold. Let $\Psi$ be a ground state of $H$. Then $a_{ \pm}^{r}(h) \Psi=0$ for any $h \in \mathbf{M}_{2}$. 
Proof. Let $H \Psi=G \Psi$. Then we see that $\left\|a_{ \pm}^{r}(h) \Psi\right\|=\lim _{t \rightarrow \pm \infty}\left\|a^{r}\left(e^{i t \omega-i t G} h\right) \Psi\right\|$ for $h \in C_{0}^{\infty}\left(\mathbb{R}^{d} \backslash \mathcal{P}\right)$. Let $\Phi=f \otimes a^{\dagger r_{1}}\left(f_{1}\right) \ldots a^{\dagger r_{n}}\left(f_{n}\right) \Omega$. Here $f \in L^{2}\left(\mathbb{R}^{d N}\right)$, $f_{1}, . ., f_{n} \in C^{\infty}\left(\mathbb{R}^{d}\right)$. Then

$$
a^{r}\left(e^{i t \omega-i t G} h\right) \Phi=\sum_{j=1}^{n}\left(e^{-i t \omega+i t G} \bar{h}, f_{j}\right) f \otimes a^{\dagger r_{1}}\left(f_{1}\right) \ldots a^{\dagger r_{j}}\left(f_{j}\right) \ldots a^{\dagger r_{n}}\left(f_{n}\right) \Omega,
$$

where ${ }^{-}$denotes omitting the terms under the symbol. Since, by [39, Theorem XI.19], with a positive constant $A\left|\left(e^{-i t \omega+i t G} \bar{h}, f_{j}\right)\right|<A /(1+|t|)^{(d-1) / 2}$, we have $\lim _{t \rightarrow \infty}\left\|a^{r}\left(e^{i t \omega-i t G} h\right) \Phi\right\|=0$. The set of the finite linear sums of $\Phi$ 's is dense in $\mathcal{H}$. Hence (4.14) gives us that, for $\Phi \in D\left(|H|^{1 / 2}\right)$ and $h \in \mathbf{M}_{2}$,

$$
\lim _{t \rightarrow \infty}\left\|a^{r}\left(e^{i t \omega-i t G} h\right) \Phi\right\|=0 \text {. }
$$

This ends the proof.

4.3. Absolutely continuous spectrum. In this subsection we specify the absolutely continuous spectrum of $H$. If $\Omega_{g}$ is a ground state of $H$, then, since $\Omega_{g} \in C^{\infty}(H)$, from Lemma 4.11, it follows that $\Omega_{g} \in D\left(\Pi_{j=1}^{n} a_{ \pm}^{\dagger r_{j}}\left(h_{j}\right)\right)$ and, $a_{ \pm}^{\dagger r_{1}}\left(h_{1}\right) a_{ \pm}^{\dagger r_{2}}\left(h_{2}\right) \ldots a_{ \pm}^{\dagger r_{n}}\left(h_{n}\right) \Omega_{g} \in D(H)$ for $h_{j} \in \bigcap_{k=-1}^{2 n} \mathcal{M}_{k}$. Then, in particular, by Lemmas 4.8 and 4.12, we see that, for $f \in \mathbf{M}_{4}, a_{ \pm}^{\dagger r_{1}}\left(h_{1}\right) a_{ \pm}^{\dagger r_{2}}\left(h_{2}\right) \ldots a_{ \pm}^{\dagger r_{n}}\left(h_{n}\right) \Omega_{g} \in$ $D\left(a^{r}(f)\right)$ and

$$
\begin{aligned}
& a^{r}(f) a_{ \pm}^{\dagger r_{1}}\left(h_{1}\right) a_{ \pm}^{\dagger r_{2}}\left(h_{2}\right) \ldots a_{ \pm}^{\dagger r_{n}}\left(h_{n}\right) \Omega_{g} \\
& \left.\quad=\sum_{j=1}^{n}\left(\bar{f}, h_{j}\right) \delta_{r, r_{j}} a_{ \pm}^{\dagger r_{1}}\left(h_{1}\right) \ldots \widehat{a_{ \pm}^{\dagger r_{j}}\left(h_{j}\right.}\right) \ldots a_{ \pm}^{\dagger r_{n}}\left(h_{n}\right) \Omega_{g} .
\end{aligned}
$$

For a normalized ground state $\Omega_{g}$, i.e., $\left\|\Omega_{g}\right\|=1$, of $H$ we define an asymptotic incoming Fock space, $\mathcal{F}_{-}:=\mathcal{F}_{-}\left(\Omega_{g}\right)$, and an asymptotic outgoing Fock space, $\mathcal{F}_{+}:=\mathcal{F}_{+}\left(\Omega_{g}\right)$, by

$$
\mathcal{F}_{ \pm}:=\overline{\left\{a_{ \pm}^{\dagger r_{1}}\left(h_{1}\right) \ldots a_{ \pm}^{\dagger r_{n}}\left(h_{n}\right) \Omega_{g}, \Omega_{g} \mid h_{j} \in \bigcap_{k=-1}^{2 n} \mathcal{M}_{k}, r_{j}=1, \ldots, d-1, j=1, \ldots, n, n \in \mathbb{N}\right\}}
$$

Here $\overline{\{\ldots\}}$ denotes the closed linear hull of $\{\ldots\}$. We have $\mathcal{F}_{ \pm}=\bigoplus_{n=0}^{\infty} \mathcal{F}_{ \pm}^{(n)}$, where

$$
\begin{aligned}
\mathcal{F}_{ \pm}^{(n)} & :=\overline{\left\{a_{ \pm}^{\dagger r_{1}}\left(h_{1}\right) \ldots a_{ \pm}^{\dagger r_{n}}\left(h_{n}\right) \Omega_{g} \mid h_{j} \in \bigcap_{k=-1}^{2 n} \mathcal{M}_{k}, r_{j}=1, \ldots, d-1, j=1, \ldots, n\right\}}, \\
\mathcal{F}_{ \pm}^{(0)} & :=\left\{\alpha \Omega_{g} \mid \alpha \in \mathbb{C}\right\} .
\end{aligned}
$$

We define wave operators $W_{ \pm}^{(n)}: \mathcal{F}^{(n)} \rightarrow \mathcal{F}_{ \pm}^{(n)}, n \geq 0$, by

$$
\begin{gathered}
W_{ \pm}^{(0)} \Omega:=\Omega_{g}, \quad W_{ \pm}^{(n)} a^{\dagger r_{1}}\left(h_{1}\right) \ldots a^{\dagger r_{n}}\left(h_{n}\right) \Omega:=a_{ \pm}^{\dagger r_{1}}\left(h_{1}\right) \ldots a_{ \pm}^{\dagger r_{n}}\left(h_{n}\right) \Omega_{g}, \quad n \geq 1 \\
h_{j} \in \bigcap_{k=-1}^{2 n} \mathcal{M}_{k}, \quad r_{j}=1, \ldots, d-1, \quad j=1, \ldots, n .
\end{gathered}
$$

Define an incoming wave operator, $W_{-}$, and an outgoing wave operator, $W_{+}$, by

$$
W_{ \pm}:=\bigoplus_{n=0}^{\infty} W_{ \pm}^{(n)}: \mathcal{F} \rightarrow \mathcal{F}_{ \pm}
$$


Lemma 4.13. Assume that Hypothesis 1, 2, 3 and 4 hold. Then $W_{ \pm}$can be uniquely extended to unitary operators of $\mathcal{F}$ to $\mathcal{F}_{ \pm}$, respectively.

Proof. It is sufficient to show that $W_{ \pm}^{(n)}$ can be extended to unitary operators of $\mathcal{F}^{(n)}$ to $\mathcal{F}_{ \pm}^{(n)}$, respectively. By 4.26), the commutation relations of $a_{ \pm}^{\sharp r}(h)$ show that, for $\Psi=a^{\dagger r_{1}}\left(h_{1}\right) \ldots a^{\dagger r_{n}}\left(h_{n}\right) \Omega, h_{j} \in \bigcap_{k=-1}^{2 n} \mathcal{M}_{k}$,

$$
\begin{aligned}
& \left(W_{ \pm}^{(n)} a^{\dagger s_{1}}\left(h_{1}\right) \ldots a^{\dagger s_{n}}\left(h_{n}\right) \Omega, W_{ \pm}^{(n)} a^{\dagger r_{1}}\left(f_{1}\right) ; a^{\dagger r_{n}}\left(f_{n}\right) \Omega\right)_{\mathcal{H}} \\
& =\left(a_{ \pm}^{\dagger s_{1}}\left(h_{1}\right) \ldots a_{ \pm}^{\dagger s_{n}}\left(h_{n}\right) \Omega_{g}, a_{ \pm}^{\dagger r_{1}}\left(f_{1}\right) \ldots a_{ \pm}^{\dagger r_{n}}\left(f_{n}\right) \Omega_{g}\right)_{\mathcal{H}} \\
& =2^{-n} \sum_{\pi \in \mathcal{P}_{n}}\left(h_{1}, f_{\pi(1)}\right) \ldots\left(h_{n}, f_{\pi(n)}\right) \delta_{s_{1}, r_{\pi(1)}} \ldots \delta_{s_{n}, r_{\pi(n)}} \\
& =\left(a^{\dagger s_{1}}\left(h_{1}\right) \ldots a^{\dagger s_{n}}\left(h_{n}\right) \Omega, a^{\dagger r_{1}}\left(f_{1}\right) \ldots a^{\dagger r_{n}}\left(f_{n}\right) \Omega\right)_{\mathcal{F}},
\end{aligned}
$$

where $\mathcal{P}_{n}$ denotes the set of the permutation of $n$-elements. The set of the finite linear sums of $\Psi$ 's and $W_{ \pm}^{(n)} \Psi$ 's form a dense set in $\mathcal{F}^{(n)}$ and $\mathcal{F}_{ \pm}^{(n)}$, respectively. Hence $W_{ \pm}^{(n)}$ can be uniquely extended to unitary operators from $\mathcal{F}^{(n)}$ to $\mathcal{F}_{ \pm}^{(n)}$, respectively.

Let us state the main theorem in this section.

Theorem 4.14. Assume that Hypothesis 1, 2, 3 and 4 hold. Then it follows that $\sigma(H)=\sigma_{a c}(H)=[G(H), \infty)$.

Proof. By Lemma 4.9] we see that, for $h_{j} \in \bigcap_{k=-1}^{2 n} \mathcal{M}_{k}$,

$$
e^{i t H} a_{ \pm}^{\dagger r_{1}}\left(h_{1}\right) \ldots a_{ \pm}^{\dagger r_{n}}\left(h_{n}\right) \Omega_{g}=a_{ \pm}^{\dagger r_{1}}\left(e^{i t \omega} h_{1}\right) \ldots a_{ \pm}^{\dagger r_{n}}\left(e^{i t \omega} h_{n}\right) e^{i t G(H)} \Omega_{g}
$$

Hence we conclude that $e^{i t H}$ leaves $\mathcal{F}_{ \pm}$invariant. This means that $H$ is reduced by $\mathcal{F}_{ \pm}$, then $H$ is also reduced by $\mathcal{F}_{ \pm}{ }^{\perp}$. Thus, corresponding to the decompositions $\mathcal{H}=\mathcal{F}_{ \pm} \oplus \mathcal{F}_{ \pm}{ }^{\perp}$, we have $H=H\left\lceil_{\mathcal{F}_{ \pm}} \oplus H\left\lceil_{\mathcal{F}_{ \pm}}{ }^{\perp}\right.\right.$. We also see that, for $h_{j} \in \bigcap_{k=-1}^{2 n} \mathcal{M}_{k}$,

$$
\begin{gathered}
W_{ \pm} e^{i t H_{\mathrm{f}}} a^{\dagger r_{1}}\left(h_{1}\right) \ldots a^{\dagger r_{n}}\left(h_{n}\right) \Omega=a_{ \pm}^{\dagger r_{1}}\left(e^{i t \omega} h_{1}\right) \ldots a_{ \pm}^{\dagger r_{n}}\left(e^{i t \omega} h_{n}\right) \Omega_{g} \\
=e^{i t(H-G(H))} a_{ \pm}^{\dagger r_{1}}\left(h_{1}\right) \ldots a_{ \pm}^{\dagger r_{n}}\left(h_{n}\right) \Omega_{g} \\
=e^{i t(H-G(H))} W_{ \pm} a^{\dagger r_{1}}\left(h_{1}\right) \ldots a^{\dagger r_{n}}\left(h_{n}\right) \Omega .
\end{gathered}
$$

Thus $W_{ \pm} e^{i t\left(H_{\mathrm{f}}+G(H)\right)}=e^{i t H} W_{ \pm}$. Since $H$ is reduced by $\mathcal{F}_{ \pm}$, $e^{i t H} \Gamma_{\mathcal{F}_{ \pm}}$are oneparameter unitary groups in $\mathcal{F}_{ \pm}$and their self-adjoint generators are $H\left\lceil\mathcal{F}_{+}\right.$, respectively. By Lemma 4.13, $W_{ \pm}$are unitary operators from $\mathcal{F}$ to $\mathcal{F}_{ \pm}$, respectively. Then we see that $W_{ \pm}$map $D\left(H_{\mathrm{f}}\right)$ onto $D\left(H\left\lceil\mathcal{F}_{ \pm}\right)=D(H) \cap \mathcal{F}_{ \pm}\right.$and implement

$$
H_{\mathrm{f}}+G(H)=W_{ \pm}^{*}\left(H\left\lceil\mathcal{F}_{ \pm}\right) W_{ \pm},\right.
$$

respectively. By Lemma 4.13, $W_{ \pm}$also induce

$$
\mathcal{H}=\mathcal{F}_{ \pm} \oplus \mathcal{F}_{ \pm}^{\perp} \cong \mathcal{F} \oplus \mathcal{F}_{ \pm}^{\perp}
$$

respectively. By (4.27), under (4.28), we have $H \cong\left\{H_{\mathrm{f}}+G(H)\right\} \oplus H\left\lceil_{\mathcal{F}_{ \pm}{ }^{\perp}}\right.$. Since $\sigma\left(H_{\mathrm{f}}+G(H)\right)=\sigma_{\mathrm{ac}}\left(H_{\mathrm{f}}+G(H)\right)=[G(H), \infty)$, this implies $[G(H), \infty) \subset \sigma_{\mathrm{ac}}(H)$. Hence we get the desired results. 


\section{NONRELATIVISTIC PARTICLES WITH SPIN}

In the case where each particle has spin, we can also get the same results as those of the spinless case. For simplicity we assume that $d=3$. The Hilbert space of the system is $\mathcal{H}_{\sigma}:=\mathbb{C}^{2} \otimes L^{2}\left(\mathbb{R}^{d N}\right) \otimes \mathcal{F}$ and its Hamiltonian is defined by

$$
H_{\sigma}:=H_{\sigma}(\hat{\rho}):=\frac{1}{2} \sum_{j=1}^{N}\left\{\sigma^{j} \otimes\left(P^{j} \otimes I-e A\left(x^{j}\right)\right)\right\}^{2}+\left(V+V_{\mathrm{osc}}\right) \otimes I+I \otimes H_{\mathrm{f}} .
$$

Here $\sigma^{j}:=\left(\sigma_{1}, \sigma_{2}, \sigma_{3}\right), j=1, \ldots, N$, and

$$
\sigma_{1}:=\left(\begin{array}{cc}
0 & 1 \\
1 & 0
\end{array}\right), \quad \sigma_{2}:=\left(\begin{array}{cc}
0 & -i \\
i & 0
\end{array}\right), \quad \sigma_{3}:=\left(\begin{array}{cc}
1 & 0 \\
0 & -1
\end{array}\right) .
$$

We immediately see that $H_{\sigma}=H_{0}+e H_{\mathrm{I}}+e^{2} H_{\mathrm{II}}+e(1 / 2) \sum_{j=1}^{N} \sigma^{j} \otimes B\left(x^{j}\right)$, where

$$
\begin{aligned}
B_{\mu}\left(x^{j}\right):=\sum_{r=1}^{2} \int \frac{d k}{\sqrt{2 \omega(k)}}\left\{\hat{\rho}(-k)\left(i k \times e^{r}(k)\right)_{\mu} e^{i k x^{j}} a^{r}(k)\right. \\
\left.+\hat{\rho}(k)\left(-i k \times e^{r}(k)\right)_{\mu} e^{-i k x^{j}} a^{\dagger r}(k)\right\} .
\end{aligned}
$$

Note that $(1 / 2) \sum_{j=1}^{N} \sigma^{j} \otimes B\left(x^{j}\right)$ is relatively bounded with respect to $H_{0}$.

Remark 5.1. (1) Assume that $\hat{\rho} \in \mathbf{M}_{4}, \overline{\hat{\rho}(k)}=\hat{\rho}(-k)$, and that $e$ is sufficiently small. Then $H_{\sigma}$ is self-adjoint on $D\left(H_{\sigma}\right)=D\left(H_{0}\right)$.

(2) As far as we know, it is not known whether diamagnetic inequalities for $H_{\sigma}$ hold or not [22].

We have the following theorems:

Theorem 5.2. Assume that Hypothesis 2 and 3 hold and that $e$ is sufficiently small. Then the ground states of $H_{\sigma}$ exist.

Theorem 5.3. Assume that Hypothesis 2, 3, and 4 hold and that e is sufficiently small. Then $s-\lim _{t \rightarrow \pm \infty} e^{i t H_{\sigma}} e^{-i t H_{0}}\left(I \otimes a^{\sharp r}(f)\right) e^{i t H_{0}} e^{-i t H_{\sigma}} \Psi$ exists for $f \in$ $C_{0}^{\infty}\left(\mathbb{R}^{d} \backslash \mathcal{P}\right)$ and $\Psi \in D\left(H_{\sigma}\right)$.

Theorem 5.4. Assume that Hypothesis 2, 3 and 4 hold and that $e$ is sufficiently small. Then $\sigma\left(H_{\sigma}\right)=\sigma_{a c}\left(H_{\sigma}\right)=\left[G\left(H_{\sigma}\right), \infty\right)$.

Proofs of Theorems 5.2, 5.3 and 5.4 are quite similar to those of Theorems 3.14 4.4 and 4.14 respectively.

\section{Concluding Remarks}

(S-matrix) When an $m$-photon state is sent with momentum distributions $f_{1}, \ldots, f_{m} \in \bigcap_{k=-1}^{2 m} \mathcal{M}_{k}$ polarized to directions $e^{s_{1}}, \ldots, e^{s_{m}}$, respectively, the probability amplitude for finding an outgoing $n$-photon state with momentum distributions $g_{1}, \ldots, g_{n} \in \bigcap_{k=-1}^{2 n} \mathcal{M}_{k}$ polarized to directions $e^{r_{1}}, \ldots, e^{r_{n}}$, respectively, is given by

$$
\begin{aligned}
& S\left(g_{1}, r_{1}, \ldots, g_{n}, r_{n} \mid f_{1}, s_{1}, \ldots, f_{m}, s_{m}\right) \\
& \quad:=\left(a_{+}^{\dagger r_{1}}\left(g_{1}\right) \ldots a_{+}^{\dagger r_{n}}\left(g_{n}\right) \Omega_{g}, a_{-}^{\dagger s_{1}}\left(f_{1}\right) \ldots a_{-}^{\dagger s_{m}}\left(f_{m}\right) \Omega_{g}\right) .
\end{aligned}
$$

For ground state $\Omega_{g}$ of $H$, the scattering operator may be defined by $S:=W_{-}^{*} W_{+}$. The unitarity of $S$ corresponds to whether $\mathcal{F}_{-}=\mathcal{F}_{+}$. 
(Quadratic potentials) In the proof of Lemma [3.5] it is needed that $\left(\left|x^{j}\right| \otimes I\right)\left(H_{0}-z\right)^{-1}$ and $\left(\left|x^{j}\right| \otimes H_{\mathrm{f}}^{1 / 2}\right)\left(H_{0}-z\right)^{-1}$ are bounded, and in the proof of Theorem 4.4 (4.11) and (4.12) are needed. In order to verify the above statements, it is assumed that $V_{\mathrm{ex}}=V+V_{\mathrm{osc}}$ in this paper. In [14, [26] the existence of the ground states of $H$ with Coulomb potentials is proven.

(Asymptotic fields) When $d \geq 4$, or/and the photon has a positive mass, i.e., $\omega(k)=\sqrt{k^{2}+\mu^{2}}, \mu>0$, in Theorem 4.4, we do not need that $\partial_{\nu} \hat{\rho} \in \mathcal{E}_{0}^{\infty}, \nu=$ $1, \ldots, d$, in Hypothesis 4 and that $H_{\mathrm{el}}$ has $V_{\mathrm{osc}}$. In the proof of Theorem 4.4, the cases of $d=2,3$ and the massless photon are crucial.

(Infrared cutoff) In Lemmas 3.2, 3.4, 3.5, 3.6, 3.7, 3.8, 3.10 and Proposition 3.3, we do not need $\|\hat{\rho}\|_{-3}<\infty$ in Hypothesis 3. Assumption $\|\hat{\rho}\|_{-3}<\infty$ is needed in the proof of "the overlap" (see Lemma 3.13). Physically it should be $\hat{\rho}(0)=(2 \pi)^{-d / 2} \int_{\mathbb{R}^{d}} \rho(x) d x=(2 \pi)^{-d / 2} e \neq 0$. However, in the case where $d=2,3$, $\|\hat{\rho}\|_{-3}<\infty$ implies that $\hat{\rho}(0)=0$. In [14] the existence of the ground states of $H$ is proven without $\|\hat{\rho}\|_{-3}<\infty$.

\section{ACKNOWLEDGMENT}

It is a pleasure to thank S. Albeverio for the hospitality of Ruhr Universität and Bonn Universität. I also thank him for bringing to my attention [1] and 3]. I thank A. Arai for pointing out some improvements of the first version of this manuscript. I am grateful to M. Hirokawa for his useful comments.

\section{REFERENCES}

[1] S.Albeverio, Scattering theory in a model of quantum fields. I, J. Math. Phys. 14, 1800-1816 (1972). MR 52:16370

[2] S.Albeverio, Scattering theory in a model of quantum fields. II, Helvetica Physica Acta 45, 303-321 (1972).

[3] S.Albeverio, An introduction to some mathematical aspects of scattering theory in models of quantum fields, Scattering theory in mathematical physics (J. A. LaVita and J. P. Marchand, eds.), 299-381 (1974).

[4] A.Amann, Ground states of a spin-boson models, Ann. Phys. 208, 414-448 (1991). MR 92k:82005

[5] A.Arai, Selfadjointness and spectrum of Hamiltonians in nonrelativistic quantum electrodynamics, J. Math. Phys. 22, 534-537 (1981). MR 82g:81013

[6] A.Arai, On a model of a harmonic oscillator coupled to a quantized, massless, scalar field I, J. Math. Phys. 22, 2539-2548 (1981). MR 83b:81032

[7] A.Arai, On a model of a harmonic oscillator coupled to a quantized, massless, scalar field II, J. Math. Phys. 22, 2549-2552 (1981). MR83b:81032

[8] A.Arai, Rigorous theory of spectra and radiation for a model in quantum electrodynamics, J. Math. Phys. 24, 1896-1910 (1983). MR 85b:81274

[9] A.Arai, A note on scattering theory in nonrelativistic quantum electrodynamics, J. Phys. A:Math. Gen. 16, 49-70 (1983). MR 84k:81124

[10] A.Arai, Perturbation of embedded eigenvalues: general class of exactly soluable models in Fock spaces, Hokkaido Math. J. 19, 1-34 (1990). MR 91c:47025

[11] A.Arai and M.Hirokawa, On the existence and uniqueness of the ground states of a generalized spin-boson model, J. Funct. Anal. 151, 455-503 (1997). MR 99b:82012

[12] H.A. Bethe, The electromagnetic shift of energy levels, Phys. Rev. 72, 339-342 (1947).

[13] V.Bach, J.Fröhlich and I.M.Sigal, Quantum electrodynamics of confined nonrelativistic particles, Adv. Math. 137, 299-395 (1998). MR 99e:81051b

[14] V.Bach, J.Fröhlich and I.M.Sigal, Spectral analysis for systems of atoms and molecules coupled to the quantized radiation field, Comm. Math. Phys. 207, 249-290 (1999). CMP 2000:05 
[15] V.Bach, J.Fröhlich, I.M.Sigal, and A.Soffer, Positive commutators and the spectrum of PauliFierz Hamiltonian of atoms and molecules, Comm. Math. Phys. 207, 557-587 (1999). MR 2000m:81046

[16] C.Fefferman, On electrons and nuclei in a magnetic field, Adv. in Math. 124, 100-153 (1996). MR 98b:81291

[17] C.Fefferman, J.Fröhlich, G.M.Graf, Stability of ultraviolet-cutoff quantum electrodynamics with non-relativistic matter, Comm. Math. Phys. 190, 309-330 (1997). MR 98k:81325

[18] J.Fröhlich, On the infrared problem in a model of scalar electrons and massless, scalar bosons, Ann. Inst. Henri Poincaré 16, 1-103 (1973). MR 51:4890

[19] J.Fröhlich, Existence of dressed one electron states in a class of persistent models, Fortschritte der Physik 22, 159-198 (1974).

[20] C.Gérard, Asymptotic completeness for the spin-boson model with a particle number cutoff, Rev. Math. Phys. 8, 549-589 (1996). MR 97j:81387

[21] J.Glimm and A.Jaffe, The $\lambda\left(\phi^{4}\right)_{2}$ quantum field theory without cutoffs:II. The field operators and approximate vacuum, Ann. Math. 91, 362-401 (1970). MR 41:1333

[22] I.Herbst, Translation invariance of $N$-particle Schrödinger operators in homogeneous magnetic fields, in Mathematical Methods and Applications of Scattering Theory, Lecture Notes in Physics 130, 169-174 (1980).

[23] F.Hiroshima, Diamagnetic inequalities for systems of nonrelativistic particles with a quantized field, Rev. Math. Phys. 8 185-203 (1996). MR 97k:81153

[24] F.Hiroshima, Functional integral representation of a model in quantum electrodynamics, Rev. Math. Phys. 9 489-530 (1997). MR 98f:81364

[25] F.Hiroshima, Ground states of nonrelativistic quantum electrodynamics I, J. Math. Phys. 40, 6209-6222 (1999). MR 2001a:81277

[26] F.Hiroshima, Ground states of nonrelativistic quantum electrodynamics II, J. Math. Phys. 41, 661-674 (2000). MR 20001a:81278

[27] F.Hiroshima, Essential self-adjointness of a translation-invariant quantum field models for arbitrary coupling constants, Comm. Math. Phys. 211, 585-613 (2000). CMP 2000:17

[28] F.Hiroshima, Point spectra and asymptotics of models coupled to a quantum field: A functional integral approach, preprint 1999.

[29] M.Hübner and H.Spohn, Spectral properties of the spin-boson Hamiltonian, Ann. Inst. Henri. Poincaré 62, 289-323 (1995). MR 96e:81249

[30] M.Hübner and H.Spohn, Radiative decay: nonperturbative approach, Rev. Math. Phys. 7, 363-387 (1995). MR 96i:81292

[31] R.H $\phi$ egh-Krohn, Asymptotic fields in some models of quantum field theory I, J. Math. Phys. 9, 2075-2080 (1968). MR 38:4116

[32] R.H $\phi$ egh-Krohn, Asymptotic fields in some models of quantum field theory II, J. Math. Phys. 10, 639-643 (1969). MR 41:3035

[33] R.H $\phi$ egh-Krohn, Asymptotic fields in some models of quantum field theory III, J. Math. Phys. 11, 185-189 (1969). MR 41:3035

[34] R.H $\phi$ egh-Krohn, On the scattering theory for quantum fields, Comm. Math. Phys. 18, 109-126 (1970).

[35] R.H $\phi$ egh-Krohn, Boson fields with bounded interaction densities, Comm. Math. Phys. 17, 179-193 (1970).

[36] R.H $\phi$ egh-Krohn, Boson fields under a general class of cut-off interactions with bounded interaction densities, Comm. Math. Phys. 12, 216-225 (1969).

[37] M. Lieb and M. Loss, Self-energy of electrons in non-perturbative QED, Differential equations and mathematical physics, 279-293, AMS/IP Stud. Adv. Math., 16, Amer. Math. Soc., Providence, RI, 2000. CMP 2000:14

[38] B.Simon and $\mathrm{H} \phi$ egh-Krohn, Hypercontractive semigroup and two dimensional self-coupled Bose fields, J. Funct. Anal. 9 121-180 (1972). MR 45:2528

[39] M.Reed and B.Simon, Method of Modern Mathematical Physics Vol. II, Academic Press, New York (1975). MR 56:12429b

[40] M.Reed and B.Simon, Method of Modern Mathematical Physics Vol.III, Academic Press, New York (1979). MR 80m:81085

[41] H. Spohn, Ground state(s) of the spin-boson Hamiltonian, Comm. Math. Phys. 123, 277-304 (1989). MR 90g:82020 
[42] H.Spohn, Asymptotic completeness for Rayleigh scattering, J. Math. Phys. 38, 2281-2296 (1997). MR 98c:81242

[43] T.A.Welton, Some observable effects of the quantum mechanical fluctuations of the electromagnetic field, Phys. Rev. 74, 1157-1169 (1948).

Institute of Applied Mathematics, University of Bonn, Wegelerstrasse 6, D53115 Bonn, Germany

Current address: Department of Mathematics and Physics, Setsunan University, Ikeda-nakamachi 17-8, 572-8508, Osaka, Japan

E-mail address: hiroshima@mpg.setsunan.ac.jp 